Math. Model. Nat. Phenom.

Vol. 9, No. 4, 2014, pp. 65-87

DOI: $10.1051 / \mathrm{mmnp} / 20149405$

\title{
A Numerical Method for the Controls of the Heat Equation
}

\author{
I. F. Bugariu ${ }^{1}$, S. Micu ${ }^{1 *}$ \\ ${ }^{1}$ Facultatea de Matematica si Stiinte ale Naturii, Universitatea din Craiova, 200585, Romania
}

\begin{abstract}
This work is devoted to analyze a numerical scheme for the approximation of the linear heat equation's controls. It is known that, due to the regularizing effect, the efficient computation of the null controls for parabolic type equations is a difficult problem. A possible cure for the bad numerical behavior of the approximating controls consists of adding a singular perturbation depending on a small parameter $\varepsilon$ which transforms the heat equation into a wave equation. A space discretization of step $h$ leads us to a system of ordinary differential equations. The aim of this paper is to show that there exists a sequence of exact controls of the corresponding perturbed semi-discrete systems which converges to a control of the original heat equation when both $h$ (the mesh size) and $\varepsilon$ (the perturbation parameter) tend to zero.
\end{abstract}

Keywords and phrases: heat equation, null controllability, moment problem, biorthogonals, singular perturbation, finite difference method

Mathematics Subject Classification: 93B05, 35K05, 65N06, 30E05

\section{Introduction}

Let $\Omega$ be a bounded open smooth set from $\mathbb{R}^{n}$ and $\Gamma_{0}$ be a nonempty open part of its boundary. The following boundary null-controllability property for the linear heat equation is know to hold:given any $T>0$ and $u^{0} \in L^{2}(\Omega)$ there exists a control $v \in L^{2}\left((0, T) \times \Gamma_{0}\right)$ such that the solution of the heat equation

$$
\begin{cases}u^{\prime}(t, x)-\Delta u(t, x)=0 & (t, x) \in(0, T) \times \Omega \\ u(t, x)=0 & (t, x) \in(0, T) \times\left(\partial \Omega \backslash \Gamma_{0}\right) \\ u(t, x)=v(t) & (t, x) \in(0, T) \times \Gamma_{0} \\ u(0, x)=u^{0}(x) & x \in \Omega,\end{cases}
$$

satisfies

$$
u(T, x)=0 \quad(x \in \Omega) .
$$

In the one dimensional case, the controllability problem (1.1)-(1.2) is known to be solvable since the seventies: we refer to the early contributions $[5,6]$ for proofs based on spectral methods. For more recent and general results, based on duality arguments and Carlemman type estimates, we refer to [7,13]. In these articles, the controllability property is reduced to a suitable observability inequality for the adjoint system. Moreover, in the spirit of the celebrated Hilbert Uniqueness Method (HUM) introduced by J.-L.

\footnotetext{
${ }^{*}$ Corresponding author. E-mail: sd_micu@yahoo.com
} 
Lions [14], a duality method leads to a practical way of obtaining controls of minimal $L^{2}$-norm. Indeed, these controls are given by a solution of the homogeneous adjoint equation which minimizes a suitable quadratic cost functional. Since we shall use this approach in our numerical computations, let us briefly describe it. Given $T>0$ and $\varphi^{0} \in L^{2}(\Omega)$ we consider the backward heat equation

$$
\begin{cases}-\varphi^{\prime}-\Delta \varphi(t, x)=0 & (t, x) \in(0, T) \times \Omega \\ \varphi(t, x)=0 & (t, x) \in(0, T) \times\left(\partial \Omega \backslash \Gamma_{0}\right) \\ \varphi(t, x)=0 & (t, x) \in(0, T) \times \Gamma_{0} \\ \varphi(T, x)=\varphi^{0}(x) & x \in \Omega .\end{cases}
$$

In view of the regularizing properties of the heat equation, the map

$$
\varphi^{0} \longrightarrow \int_{0}^{T} \int_{\Gamma_{0}}\left|\frac{\partial \varphi}{\partial \nu}\right|^{2}(t, x) d x d t
$$

which is well defined and continuous in some Sobolev space (for instance $H_{0}^{1}(\Omega)$ ), by unique continuation, is a norm in $L^{2}(\Omega)$ (see, for instance, [19] and the references therein). We define the Hilbert space $\mathcal{H}$ as the completion of $L^{2}(\Omega)$ with respect to norm (1.4). Now, we introduce the functional $\mathcal{J}: \mathcal{H} \rightarrow \mathbb{R}$ given by

$$
\mathcal{J}\left(\varphi^{0}\right)=\frac{1}{2} \int_{0}^{T} \int_{\Gamma_{0}}\left|\frac{\partial \varphi}{\partial \nu}\right|^{2}(t, x) d x d t-\int_{\Omega} y^{0}(x) \varphi(0, x) d x,
$$

where $\varphi$ is the solution of (1.3) with initial data $\varphi^{0}$.

For all $y^{0} \in L^{2}(\Omega), \mathcal{J}$ is coercive in $\mathcal{H}$ and has a unique minimizer $\widehat{\varphi}^{0} \in \mathcal{H}$. Moreover, the solution $\widehat{\varphi}$ of (1.3) with initial data $\widehat{\varphi}^{0}$ gives the control $v=\left.\frac{\partial \widehat{\varphi}}{\partial \nu}\right|_{\Gamma_{0}}$ of minimal $L^{2}\left((0, T) \times \Gamma_{0}\right)$-norm of (1.1) (see, for instance [19]). Hence, a control for (1.1) is obtained by minimizing the functional $\mathcal{J}$. Since there exist several efficient numerical methods for minimization processes, the duality method described above became a serious candidate to provide the numerical approximation of the controls for (1.1).

A first intent to use these ideas may be found in the seminal article of Carthel-Glowinski-Lions [4]. This paper studies the numerical implementation of the Hilbert Uniqueness Method for solving exact and approximate boundary controllability problems for the heat equation by reducing them to a minimization problem similar to the one described above. However, the numerical experiments have shown that the efficient computing of the null controls for the heat equation is a difficult problem. The very weak coercivity of the functionals under consideration and the low regularity of the minimizers make the approximation problem exponentially ill-posed and the functional framework far from being well adapted to standard techniques in numerical analysis (see [20,21,23] for analyzes of these problems). For these reasons, how to construct robust numerical approximations of exact null controls for parabolic systems remains a challenging problem.

Since then, many alternative methods have been proposed and analyzed. For instance, in [3] the controls of the heat equation are found by minimizing a cost functional with weights that blows up near the control time $T$. The weights reinforce the controllability requirement and regularize the behavior of the control near $t=T$. In [22] a least squares type method is analyzed. Instead of working with solutions of the underlying state equation, and looking for one that may comply with the final desired state, they consider a suitable class of functions complying with required initial, boundary, and final conditions, and seek one of those that is a solution of the state equation. Finally, in [23] a numerical version of the so-called transmutation method is developed. This approach allows writing the control of a heat process in terms of the corresponding control of the associated wave process, by means of a "time convolution" with a one-dimensional controlled fundamental heat solution. In spite of this interesting studies, there are still relatively few results of convergence in the literature. We mention here the recent paper [2] in which special Carlemman estimates are used to obtain uniform observability inequalities for semi-discrete semi-linear parabolic equations. 
Regularization can also be performed by modifying the heat equation with the aim to restore the stability of the corresponding backward system. This approach is considered in detail in [12] in the framework of optimal control theory by introducing the so-called quasi-reversibility methods and in $[15,16]$ in the context of controllability. In this way, instead of (1.1), the null controllability of the following damped, singularly perturbed, wave equation is considered:

$$
\begin{cases}\varepsilon u^{\prime \prime}(t, x)+u^{\prime}(t, x)-\Delta u(t, x)=0 & (t, x) \in(0, T) \times \Omega \\ u(t, x)=0 & (t, x) \in(0, T) \times\left(\partial \Omega \backslash \Gamma_{0}\right) \\ u(t, x)=v_{\varepsilon}(t) & (t, x) \in(0, T) \times \Gamma_{0} \\ u(0, x)=u^{0}(x), u^{\prime}(0, x)=0 & x \in \Omega,\end{cases}
$$

In (1.5), $\varepsilon$ is a small parameter devoted to tend to zero. It can be shown that, given any $T>0,(1.5)$ is uniformly (with respect to $\varepsilon$ ) controllable in time $T$ : the control of minimal $L^{2}$-norm corresponding to (1.5) converges, as $\varepsilon$ goes to zero, to the minimal $L^{2}$-norm control of (2.1). We refer the interested reader to [18] in which the interior control case is studied.

The aim of this paper is to show that the same property holds for the semi-discrete version of (1.5), at least in the one dimensional case. More precisely, we discretize in space (1.5) and we show that, for any $T>0$ and any discrete initial data, there exists a sequence of exact controls of the system which converges to a control of (1.1), when the mesh size $h$ and the perturbation parameter $\varepsilon$ go simultaneously to zero. To show this we use a technique which is different from the one in [18] and it is close to the spectral approach from [5,6]. Firstly, we reduce the controllability problem to a moment problem. Nextly, we solve this problem by constructing a biorthogonal sequence to the family of complex exponential functions $\left(e^{\lambda_{j} t}\right)_{j}$, where $\lambda_{j}$ are the eigenvalues of the matriceal operator corresponding to the discrete version of (1.5). By a careful estimate of the biorthogonal sequence we are able to show the existence of a sequence of uniformly bounded discrete controls and to deduce the desired convergence result. The perturbation parameter $\varepsilon$ will depend on $h$, the mesh size. We shall provide explicit bounds for the smallness of the parameter $\varepsilon$ with respect to $h$ which ensure this convergence. A second step, and a very interesting one, in the analysis of (1.5) would be to study the regularity of the minimizers obtained by applying to (1.5) the duality method explained before. Even in the one dimensional case, this study is much more complicated than the one in [21]. Indeed, the complex nature of the spectrum of the differential operator corresponding to (1.5) introduces new difficulties in the study of the associated Gramm matrix. This problem remains open.

Let us finally remark that this singular perturbation method has been used before in the context of ill-posed parabolic problems. Indeed, in [26] a similar idea is used to approximate numerically an ill-posed inverse heat problem. There the author first introduces a regularizing second time derivative term and then reverses the space and time variables. This leads to a well-posed hyperbolic problem whose solution is close to the desired one.

The rest of the paper is organized as follows. In Section 2 we present the continuous one-dimensional boundary control problem for the heat equation and we transform it into a moment problem. Section 3 is devoted to study some of the controllability and spectral properties of the semi-discrete system corresponding to the perturbed equation. In Section 4 we introduce the discrete moment problem and in Section 5 we construct a uniformly bounded biorthogonal sequence to a particular family of exponential functions. Finally, Section 6 is devoted to show the convergence properties of the discrete controls and Section 7 to show several numerical computations.

\section{Null-controllability of the continuous heat equation}

In this section we recall some well-known properties concerning the boundary null-controllability problem for the continuous 1-D linear heat equation and we characterize the control by using the moment theory. More precisely, given any $T>0$ and $u^{0} \in L^{2}(0,1)$ we look for a control $v \in L^{2}(0, T)$ such that the 
solution of the heat equation

$$
\begin{cases}u^{\prime}(t, x)-u_{x x}(t, x)=0 & (t, x) \in(0, T) \times(0,1) \\ u(t, 0)=0 & t \in(0, T) \\ u(t, 1)=v(t) & t \in(0, T) \\ u(0, x)=u^{0}(x) & x \in(0,1),\end{cases}
$$

satisfies

$$
u(T, x)=0 \quad(x \in(0,1)) .
$$

If for any $u^{0} \in L^{2}(0,1)$ there exists a control $v \in L^{2}(0, T)$ for $(2.1)-(2.2)$, we say that the problem (2.1) is null-controllable in time $T$.

In order to provide a control $v$ for $(2.1)-(2.2)$ we deduce an equivalent moment problem whose solution is given in terms of an explicit biorthogonal sequence to the family of exponential functions $\left(e^{n^{2} \pi^{2} t}\right)_{n \in \mathbb{N}^{*}}$. We recall that, given $\mathcal{I} \subset \mathbb{Z}^{*},\left(\theta_{m}\right)_{m \in \mathcal{I}} \subset L^{2}(a, b)$ is a biorthogonal sequence to the family of exponential functions $\left(e^{\lambda_{n} t}\right)_{n \in \mathcal{I}}$ in $L^{2}(a, b)$ if and only if

$$
\int_{a}^{b} \theta_{m}(t) e^{\bar{\lambda}_{n} t} d t=\delta_{m n} \quad(n, m \in \mathcal{I}) .
$$

The moments method was used by Fattorini and Russell in the pioneering articles [5,6] to solve one dimensional control problems for parabolic type equations even more general than (2.1). Since then, this method has been widely used to solve a large variety of linear control problem. We refer to $[1,11,25]$ for a detailed discussion of the subject.

It follows easily that (2.1) is null-controllable in time $T$ if, and only if, for every initial datum $u^{0} \in$ $L^{2}(0,1)$ with the Fourier expansion

$$
u^{0}(x)=\sum_{n \geq 1} a_{n} \sqrt{2} \sin (\pi n x)
$$

there exists a solution $v \in L^{2}(0, T)$ of the following moment problem:

$$
(-1)^{n} \int_{0}^{T} v(t) e^{n^{2} \pi^{2} t} d t=\frac{1}{\sqrt{2} n \pi} a_{n} \quad\left(n \in \mathbb{N}^{*}\right) .
$$

To fully understand the method that we use in this paper, let us briefly describe the steps needed to obtain a solution for (2.5). Firstly, we construct a biorthogonal sequence $\left(\zeta_{m}\right)_{m \in \mathbb{N}^{*}}$ in $L^{2}(0, T)$ to the family $\left(e^{n^{2} \pi^{2} t}\right)_{n \in \mathbb{N}^{*}}$ which verifies the following estimate

$$
\left\|\zeta_{m}\right\|_{L^{2}(0, T)} \leq M e^{\widetilde{\omega} m} \quad\left(m \in \mathbb{N}^{*}\right)
$$

where $M$ and $\widetilde{\omega}$ are two positive constants. The existence of such sequence $\left(\zeta_{m}\right)_{m \in \mathbb{N}^{*}}$ is a consequence of Müntz's Theorem (see $[5,6,19]$ for full details). Once we have the biorthogonal sequence $\left(\zeta_{m}\right)_{m \in \mathbb{N}^{*}}$, an explicit solution of $(2.5)$ is given through the formula

$$
v(t)=\sum_{m \geq 1}(-1)^{m} \frac{a_{m}}{\sqrt{2} m \pi} e^{-m^{2} \pi^{2} T} \zeta_{m}(t) \quad(t \in(0, T)) .
$$

Note that the absolute convergence in $L^{2}(0, T)$ of the right hand side of $(2.7)$ is ensured by estimate (2.6) and the fact that $u^{0} \in L^{2}(0,1)$. Consequently, this method allows us to prove that, for any time $T>0$ and initial data (2.4), the moment problem (2.5) has at least a solution and the null-controllability property of $(2.1)$ holds. 


\section{The discrete problem}

In this section we study the controllability properties of a semi-discrete version of (2.1) by using the method described above. Let us consider $N \in \mathbb{N}^{*}$, a step $h=\frac{1}{N+1}$ and an equidistant mesh of the interval $(0,1), 0=x_{0}<x_{1}<\ldots<x_{N}<x_{N+1}=1$ with $x_{j}=j h, 0 \leq j \leq N+1$. The classical central finite-difference approximation of the space derivates leads to the following semi-discretization of (2.1)

$$
\begin{cases}u_{j}^{\prime}(t)-\frac{u_{j+1}(t)+u_{j-1}(t)-2 u_{j}(t)}{h^{2}}=0 & 1 \leq j \leq N, t \in(0, T) \\ u_{0}(t)=0 & t \in(0, T) \\ u_{N+1}(t)=v_{h}(t) & t \in(0, T) \\ u_{j}(0)=u_{j}^{0} & 1 \leq j \leq N .\end{cases}
$$

System (3.1) consists of $N$ linear equation with $N$ unknowns $u_{1}, u_{2}, \ldots, u_{N}$. In other words, $u_{j}(t)$ approximates $u\left(t, x_{j}\right)$, the solution of $(2.1)$, if $\left(u_{j}^{0}\right)_{0 \leq j \leq N+1}$ is an approximation of the initial datum in (2.1). In fact, we shall choose

$$
u_{j}^{0}=u^{0}(j h) \quad(0 \leq j \leq N+1) .
$$

It is not difficult to see that equation (3.1) has the following controllability property: given $T>0$ and $\left(u_{j}^{0}\right)_{1 \leq j \leq N} \in \mathbb{C}^{N}$, there exists a control $v_{h} \in L^{2}(0, T)$ such that the corresponding solution $\left(u_{j}\right)_{1 \leq j \leq N}$ of (3.1) verifies

$$
u_{j}(T)=0 \quad(1 \leq j \leq N) .
$$

The controllability problem for (3.1) was studied in [17], where the convergence of the controls $\left(v_{h}\right)_{h>0}$ to a control $v$ of (2.1) is proved. However, as it was remarked in [23], the efficient numerical computation of controls with the above discretization by the duality method described above is a very delicate issue. This is mainly due to the fact that we need to solve the adjoint system of (3.1) with very irregular initial data which do not belong to any Sobolev space of negative exponent (see [21]). In [23] several cures are proposed. One of them will be studied in details in this paper. More precisely, we study the null-controllability of the following discretization of (2.1), different from (3.1),

$$
\left\{\begin{array}{lc}
\varepsilon u_{j}^{\prime \prime}(t)+u_{j}^{\prime}(t)-\frac{u_{j+1}(t)+u_{j-1}(t)-2 u_{j}(t)}{h^{2}}= & 01 \leq j \leq N, t \in(0, T) \\
u_{0}(t)=0 & t \in(0, T) \\
u_{N+1}(t)=v_{h}(t) & t \in(0, T) \\
u_{j}(0)=u_{j}^{0} & 1 \leq j \leq N \\
u_{j}^{\prime}(0)=u_{j}^{1} & 1 \leq j \leq N,
\end{array}\right.
$$

where the parameter $\varepsilon$ depends of $h$ and $\lim _{h \rightarrow 0} \varepsilon(h)=0$. Hence, $\varepsilon u^{\prime \prime}$ represents a singular perturbation term which eventually vanishes in the limit as $h$ tends to zero. Note that (3.4) is no longer a first order differential system as (3.1), but a second order one. As a consequence of this fact, a second initial data, $u^{1}$, is introduced. Its election will be made later on. Now, the null-controllability problem for (3.4) turns to: given any $T>0, \varepsilon>0$ and any initial data $\left(u_{j}^{0}, u_{j}^{1}\right)_{1 \leq j \leq N} \in \mathbb{C}^{2 N}$, there exists a control $v_{h} \in L^{2}(0, T)$ such that the corresponding solution $\left(u_{j}, u_{j}^{\prime}\right)_{1 \leq j \leq N}$ of $(3.4)$ verifies

$$
u_{j}(T)=u_{j}^{\prime}(T)=0 \quad(1 \leq j \leq N) .
$$

If the null-controllability problem $(3.4)-(3.5)$ has a solution $v_{h} \in L^{2}(0, T)$ for every initial data $\left(u_{j}^{0}\right)_{1 \leq j \leq N} \in \mathbb{C}^{N}$ and $\left(u_{j}^{1}\right)_{1 \leq j \leq N} \in \mathbb{C}^{N}$, we say that (3.4) is null-controllable in time $T$. In order to write (3.4) in an abstract Cauchy form, we define the matrix $A \in \mathcal{M}_{N \times N}(\mathbb{R})$

$$
A_{h}=\frac{1}{h^{2}}\left(\begin{array}{rrrrrrr}
2 & -1 & 0 & 0 & \ldots & 0 & 0 \\
-1 & 2 & -1 & 0 & \ldots & 0 & 0 \\
0 & -1 & 2 & -1 & \ldots & 0 & 0 \\
\vdots & \vdots & \ddots & \ddots & \ddots & \vdots & \vdots \\
0 & 0 & \ldots & -1 & 2 & -1 & 0 \\
0 & 0 & \ldots & 0 & -1 & 2 & -1 \\
0 & 0 & \ldots & 0 & 0 & -1 & 2
\end{array}\right) \text {. }
$$


If we denote by

$$
U_{h}^{0}=\left(\begin{array}{c}
u_{1}^{0} \\
u_{2}^{0} \\
\vdots \\
u_{N}^{0}
\end{array}\right), U_{h}^{1}=\left(\begin{array}{c}
u_{1}^{1} \\
u_{2}^{1} \\
\vdots \\
u_{N}^{1}
\end{array}\right), U_{h}(t)=\left(\begin{array}{c}
u_{1}(t) \\
u_{2}(t) \\
\vdots \\
u_{N}(t)
\end{array}\right) \quad \text { and } F_{h}(t)=\frac{1}{h^{2}}\left(\begin{array}{c}
0 \\
0 \\
\vdots \\
0 \\
v_{h}(t)
\end{array}\right)
$$

then system (3.4) may be written vectorially as follows

$$
\left\{\begin{array}{l}
\varepsilon U_{h}^{\prime \prime}(t)+U_{h}^{\prime}(t)+A_{h} U_{h}(t)=F_{h}(t) t \in(0, T) \\
U_{h}(0)=U_{h}^{0} \\
U_{h}^{\prime}(0)=U_{h}^{1}
\end{array}\right.
$$

In (3.6) we have taken into account that $u_{N+1}=v_{h}(t)$ and $u_{0}(t)=0$ for all $t \in(0, T)$.

Let us define in $\mathbb{C}^{N}$ the inner product

$$
\langle f, g\rangle=h \sum_{k=1}^{N} f_{k} \bar{g}_{k}
$$

where the vectors $f=\left(f_{k}\right)_{1 \leq k \leq N}$ and $g=\left(g_{k}\right)_{1 \leq k \leq N}$ belong to $\mathbb{C}^{N}$.

It is well known that (see, for instance, [10]) the eigenvalues of the self-adjoint matrix $A_{h}$ are given by

$$
\mu_{j}=\frac{4}{h^{2}} \sin ^{2}\left(\frac{j \pi h}{2}\right) \quad(1 \leq j \leq N)
$$

and the corresponding eigenvectors are

$$
\varphi^{j}=\sqrt{2}(\sin (n j h \pi))_{1 \leq n \leq N} \in \mathbb{R}^{N} \quad(1 \leq j \leq N) .
$$

The following property holds.

Lemma 3.1. The set of vectors $\left(\varphi^{j}\right)_{1 \leq j \leq N}$ forms an orthonormal basis in $\mathbb{C}^{N}$ with respect to the inner product given by $(3.7)$.

Proof. We notice that

$$
\left\langle\varphi^{i}, \varphi^{j}\right\rangle=2 h \sum_{n=1}^{N} \sin (n j h \pi) \sin (n i h \pi)=h \sum_{n=0}^{N}(\cos ((i-j) n h \pi)-\cos ((i+j) n h \pi)) .
$$

Nextly, for any $q \in \mathbb{Z}$, we have that

$$
\sum_{n=0}^{N} \cos (q n h \pi)= \begin{cases}N+1 & q=0 \\ \frac{1-(-1)^{q}}{2} & q \in \mathbb{Z}^{*} .\end{cases}
$$

It follows that $\left\langle\varphi^{i}, \varphi^{j}\right\rangle=\delta_{i j}$, for any $i, j \in \mathbb{N}^{*}$ and the proof of this lemma is over.

\section{The moment problem}

The aim of this section is to transform the null-controllability problem of (3.4) into a moment problem. In order to do this we introduce the following homogeneous adjoint system

$$
\left\{\begin{array}{l}
\varepsilon W_{h}^{\prime \prime}(t)-W_{h}^{\prime}(t)+A_{h} W_{h}(t)=0 t \in(0, T) \\
W_{h}(T)=W_{h}^{0} \\
W_{h}^{\prime}(T)=W_{h}^{1}
\end{array}\right.
$$

where $\left(W_{h}^{0}, W_{h}^{1}\right) \in \mathbb{C}^{2 N}$ are given. We have the following result. 
Lemma 4.1. Given any $T>0$, system (3.6) is null-controllable in time $T$ if and only if, for any $\left(U_{h}^{0}, U_{h}^{1}\right) \in \mathbb{C}^{2 N}$, there exists $v_{h} \in L^{2}(0, T)$ which verifies

$$
\int_{0}^{T} v_{h}(t) \frac{\overline{W_{h, N}(t)}}{h} d t=-\varepsilon\left\langle U_{h}^{1}, W_{h}(0)\right\rangle+\left\langle U_{h}^{0}, \varepsilon W_{h}^{\prime}(0)-W_{h}(0)\right\rangle \quad\left(\left(W_{h}^{0}, W_{h}^{1}\right) \in \mathbb{C}^{2 N}\right),
$$

where $\left(W_{h}, W_{h}^{\prime}\right)$ is the solution of (4.1) with initial data $\left(W_{h}^{0}, W_{h}^{1}\right)$.

Proof. If we multiply (3.6) by $\bar{W}_{h}$ and we integrate by parts over $(0, T)$, we obtain that $v_{h} \in L^{2}(0, T)$ is a control which leads to zero the solution of (3.6) if and only if (4.2) takes place for any $\left(W_{h}^{0}, W_{h}^{1}\right) \in \mathbb{C}^{2 N}$.

Now, let us suppose that the initial data, are given by

$$
\left(W_{h}^{0}, W_{h}^{1}\right)=\left(\sum_{j=1}^{N} b_{j h}^{0} \varphi^{j}, \sum_{j=1}^{N} b_{j h}^{1} \varphi^{j}\right)
$$

and the solution of (4.1) is written as

$$
W_{h}(t)=\sum_{j=1}^{N} b_{j h}(t) \varphi^{j}
$$

We have the following result.

Lemma 4.2. If the initial data $\left(W_{h}^{0}, W_{h}^{1}\right)$ of (4.1) are given by (4.3), then the corresponding solution of (4.1) is given by (4.4), where

$$
b_{j h}(t)=\frac{b_{j h}^{1}-\lambda_{-j} b_{j h}^{0}}{\lambda_{j}-\lambda_{-j}} e^{\lambda_{j}(t-T)}-\frac{b_{j h}^{1}-\lambda_{j} b_{j h}^{0}}{\lambda_{j}-\lambda_{-j}} e^{\lambda_{-j}(t-T)}
$$

and

$$
\lambda_{j}=\left\{\begin{array}{ll}
\frac{1+\operatorname{sgn}(j) \sqrt{1-4 \varepsilon \mu_{|j|}}}{2 \varepsilon} & \text { if } \mu_{|j|} \leq \frac{1}{4 \varepsilon} \\
\frac{1+i \operatorname{sgn}(j) \sqrt{4 \varepsilon \mu_{|j|}-1}}{2 \varepsilon} & \text { if } \mu_{|j|}>\frac{1}{4 \varepsilon},
\end{array} \quad(1 \leq|j| \leq N) .\right.
$$

The values $\left(\lambda_{j}\right)_{1 \leq|j| \leq N}$ defined by (4.6) are the eigenvalues of the operator corresponding to the adjoint problem (4.1).

Remark 4.3. For $\varepsilon \leq \frac{h^{2}}{16}$ it follows that all the eigenvalues $\lambda_{j}$ are given by

$$
\lambda_{j}=\frac{1+\operatorname{sgn}(j) \sqrt{1-4 \varepsilon \mu_{|j|}}}{2 \varepsilon} \quad(1 \leq|j| \leq N),
$$

which are purely real values. In this case the introduced perturbation is very small and problem (4.1) has a behavior similar to the continuous heat equation, (2.1).

On the other hand, if $\varepsilon>\frac{h^{2}}{16}$, we introduce the positive integer

$$
n_{\varepsilon}=\max \left\{j: 1 \leq j \leq N, \sin ^{2}\left(\frac{j \pi h}{2}\right) \leq \frac{h^{2}}{16 \varepsilon}\right\},
$$

and note that the eigenvalues $\lambda_{j}$ with $1 \leq j \leq n_{\varepsilon}$ are purely real and given by

$$
\lambda_{j}=\frac{1+\sqrt{1-4 \varepsilon \mu_{j}}}{2 \varepsilon} \text { and } \lambda_{-j}=\frac{1-\sqrt{1-4 \varepsilon \mu_{j}}}{2 \varepsilon},
$$


whereas the eigenvalues $\lambda_{j}$ with $n_{\varepsilon}<j \leq N$ are complex (with constant real part) and given by

$$
\lambda_{j}=\frac{1+i \sqrt{4 \varepsilon \mu_{j}-1}}{2 \varepsilon} \text { and } \quad \lambda_{-j}=\frac{1-i \sqrt{4 \varepsilon \mu_{j}-1}}{2 \varepsilon} .
$$

Moreover, $n_{\varepsilon}$ has the following property

$$
\frac{1}{4 \pi \sqrt{\varepsilon}} \leq n_{\varepsilon} \leq \frac{1}{4 \sqrt{\varepsilon}} .
$$

The spectrum of the operator corresponding to (4.1) has been schematically depicted in Figure 1 in the case $N=999$ and $\varepsilon=h^{1.3}$. The real eigenvalues are represented by circles whereas the complex ones, which have a constant real part, are represented by dots.

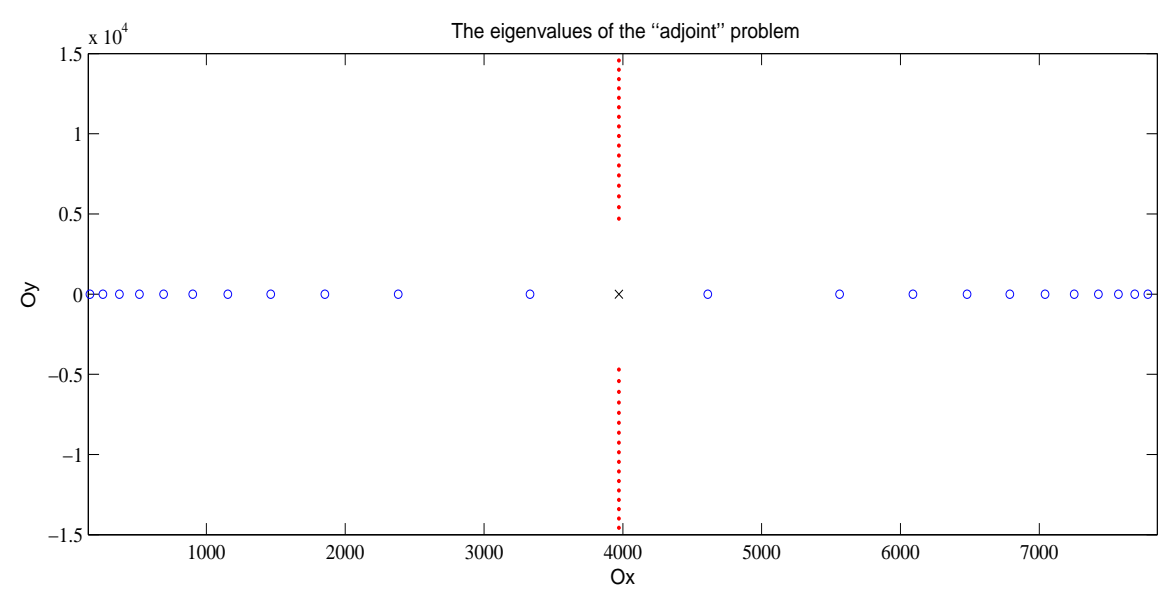

Figure 1. The eigenvalues of the operator corresponding to (4.1) for $\varepsilon=h^{1.3}$.

Remark 4.4. The eigenvalues $\lambda_{j}$, have the following elementary properties

$$
\begin{gathered}
\mu_{j}<\lambda_{-j}<2 \mu_{j} \quad\left(1 \leq j \leq n_{\varepsilon}\right), \\
\left|\lambda_{-j}\right| \leq\left|\lambda_{j}\right| \quad\left(1 \leq j \leq n_{\varepsilon}\right), \\
\left|\lambda_{j}\right|^{2}=\frac{\mu_{|j|}}{\varepsilon} \quad\left(n_{\varepsilon}<|j| \leq N\right), \\
\lambda_{j} \lambda_{-j}=\frac{\mu_{|j|}}{\varepsilon} \quad \text { and } \quad \lambda_{j}+\lambda_{-j}=\frac{1}{\varepsilon} \quad\left(j \in \mathbb{Z}^{*},|j| \leq N\right) .
\end{gathered}
$$

We have now all the ingredients needed to characterize the null-controllability property of (3.6) in terms of a moment problem.

Theorem 4.5. Let $T>0$ and $\varepsilon>0$. System (3.6) is null-controllable in time $T$ if and only if, for any initial data $\left(U_{h}^{0}, U_{h}^{1}\right) \in \mathbb{C}^{2 N}$ of form

$$
\left(U_{h}^{0}, U_{h}^{1}\right)=\left(\sum_{j=1}^{N} a_{j h}^{0} \varphi^{j}, \sum_{j=1}^{N} a_{j h}^{1} \varphi^{j}\right),
$$


there exists $v_{h} \in L^{2}(0, T)$ such that

$$
\int_{-\frac{T}{2}}^{\frac{T}{2}} v_{h}\left(t+\frac{T}{2}\right) e^{\bar{\lambda}_{j} t} d t=\frac{(-1)^{j} h e^{-\bar{\lambda}_{j} \frac{T}{2}}}{\sqrt{2} \sin (|j| \pi h)}\left(\varepsilon a_{|j| h}^{1}-\varepsilon \bar{\lambda}_{j} a_{|j| h}^{0}+a_{|j| h}^{0}\right) \quad(1 \leq|j| \leq N),
$$

where $\lambda_{j}$ are given by (4.6).

Proof. The proof of this theorem follows immediately from Lemma 4.1. Indeed, it is sufficient to verify (4.2) for the initial data $\left(W_{h}^{0}, W_{h}^{1}\right)=\left(\varphi^{|j|}, \lambda_{j} \varphi^{|j|}\right)$ for any $1 \leq|j| \leq N$. By taking into account (4.3)-(4.4), we obtain that in this case $W(t)=e^{(t-T) \lambda_{j}} \varphi^{|j|}$ and consequently

$$
\overline{W_{h, N}(t)}=(-1)^{j+1} \sqrt{2} \sin (|j| \pi h) e^{(t-T) \bar{\lambda}_{j}} .
$$

Moreover, by using (4.12) and the left hand side of (4.2) we deduce that, for $1 \leq|j| \leq N$,

$$
-\varepsilon\left\langle U_{h}^{1}, W_{h}(0)\right\rangle+\left\langle U_{h}^{0}, \varepsilon W_{h}^{\prime}(0)-W_{h}(0)\right\rangle=-\left(\varepsilon a_{|j| h}^{1}-\varepsilon \bar{\lambda}_{j} a_{|j| h}^{0}+a_{|j| h}^{0}\right) e^{-\bar{\lambda}_{j} T} .
$$

Thus, from (4.14) and (4.15) it follows that (4.13) takes place and the proof of the theorem finishes.

As mentioned before, a solution $v_{h}$ to (4.13) may be obtained from a biorthogonal sequence $\left(\theta_{m}\right)_{\substack{|m| \leq N \\ m \neq 0}}$ to the family of exponential functions $\left(e^{\lambda_{n} t}\right)_{\substack{|n| \leq N \\ n \neq 0}}$ in $L^{2}\left(-\frac{T}{2}, \frac{T}{2}\right)$. Indeed, such a solution $v_{h}$ is given by the formula

$$
v_{h}(t)=\sum_{\substack{|n| \leq N \\ n \neq 0}} \frac{(-1)^{n} h e^{-\bar{\lambda}_{n} \frac{T}{2}}}{\sqrt{2} \sin (|n| \pi h)}\left(\varepsilon a_{|n| h}^{1}-\varepsilon \bar{\lambda}_{n} a_{|n| h}^{0}+a_{|n| h}^{0}\right) \theta_{n}\left(t-\frac{T}{2}\right) \quad(t \in(0, T)) .
$$

In the following section we construct an explicit biorthogonal sequence $\left(\theta_{m}\right)_{\substack{|m| \leq N \\ m \neq 0}}$ to the family $\left(e^{\lambda_{n} t}\right)_{\substack{|n| \leq N \\ n \neq 0}}$. Moreover, since we are interested in the behavior of $v_{h}$ as $h$ tends to zero, we shall evaluate carefully the dependence of $\left(\theta_{m}\right)_{\substack{|m| \leq N \\ m \neq 0}}$ on the discrete parameters $h$ and $\varepsilon$. This analysis will allow us to pass to the limit as $h$ and $\varepsilon$ go to zero in (4.16) and to obtain a control for (2.1).

\section{Construction of a biorthogonal sequence}

As mentioned before, this section is devoted to construct and evaluate a biorthogonal sequence to the family $\left(e^{\lambda_{n} t}\right)_{\substack{|n| \leq N \\ n \neq 0}}$ in $L^{2}\left(-\frac{T}{2}, \frac{T}{2}\right)$, where $\left(\lambda_{n}\right)_{\substack{|n| \leq N \\ n \neq 0}}$ are given by (4.6). Since $\left(e^{\lambda_{n} t}\right)_{\substack{|n| \leq N \\ n \neq 0}}$ is a finite family of exponential functions, it follows immediately that it has infinitely many biorthogonal sequences in $L^{2}\left(-\frac{T}{2}, \frac{T}{2}\right)$. However we are interested not only on their existence, but also on their dependence of the parameters $h$ and $\varepsilon$. Therefore, we shall construct an explicit biorthogonal sequence and we shall evaluate its $L^{\infty}$-norm. Firstly, we construct an entire function of exponential type, $P_{m}$, and we evaluate it on the real axis. Secondly, by taking into account the estimates for $P_{m}$, we construct another entire function $M_{m}$, called multiplier, such that the product $P_{m} M_{m}$ is an entire function of arbitrarily small exponential type bounded on the real axis. This construction allows us to obtain a biorthogonal element $\theta_{m}$, through the inverse Fourier transform. Such a method was used for the first time by Paley and Wiener [24] and, in the context of controllability problems, by Fattorini and Russell [5,6]. The main difficulties in our analysis is to obtain good estimates for the behavior of $P_{m}$ on the real axis and to construct an appropriate multiplier $M_{m}$. 


\subsection{A Weierstrass product}

The aim of this subsection is to construct a Weierstrass product, vanishing in all but one of the eigenvalues (4.6), and to study his properties. For any $m \in \mathbb{Z}^{*}$, such that $|m| \leq N$ we define the product

$$
P_{m}(z)=\prod_{\substack{1 \leq|n| \leq N \\ n \neq m}}\left(1+\frac{i z}{\lambda_{n}}\right)\left(\frac{\lambda_{n}}{\lambda_{n}-\lambda_{m}}\right)
$$

The basic properties of $P_{m}$ are described in the following proposition.

Proposition 5.1. For any $m \in \mathbb{Z}^{*}$ such that $|m| \leq N, P_{m}(z)$ is an entire function of exponential type at most $\frac{(1+\pi) \sqrt{2 \varepsilon}}{2}$ with the following property

$$
P_{m}\left(i \lambda_{n}\right)=\delta_{m n} \quad(1 \leq|n| \leq N) .
$$

Proof. Property (5.2) is evident. In order to show that $P_{m}(z)$ is an entire function of exponential type, we evaluate separately the products

$$
E_{m}(z)=\prod_{\substack{1 \leq|n| \leq N \\ n \neq m}}\left(1+\frac{i z}{\lambda_{n}}\right) \quad \text { and } \quad Q_{m}=\prod_{\substack{1 \leq|n| \leq N \\ n \neq m}}\left(\frac{\lambda_{n}}{\lambda_{n}-\lambda_{m}}\right)
$$

Now, we evaluate $E_{m}(z)$ by taking into account (4.11). We have that

$$
\left|E_{m}(z)\right|=\left|\prod_{\substack{1 \leq|n| \leq N \\ n \neq m}}\left(1+\frac{i z}{\lambda_{n}}\right)\right|=\prod_{\substack{n=1 \\ n \neq m}}^{N}\left|\frac{\lambda_{n} \lambda_{-n}+i z\left(\lambda_{n}+\lambda_{-n}\right)-z^{2}}{\lambda_{n} \lambda_{-n}}\right| \leq \prod_{n=1}^{N}\left(1+\frac{|z|+\varepsilon|z|^{2}}{4 n^{2}}\right) .
$$

In order to complete the analysis we have to treat separately the following cases:

$-|z|<\frac{1}{\varepsilon}$. In this case we have that

$$
\begin{gathered}
\prod_{n=1}^{N}\left(1+\frac{|z|+\varepsilon|z|^{2}}{4 n^{2}}\right) \leq \prod_{n=1}^{N}\left(1+\frac{|z|}{2 n^{2}}\right)=\exp \left(\sum_{n=1}^{N} \ln \left(1+\frac{|z|}{2 n^{2}}\right)\right) \leq \\
\leq \exp \left(\int_{0}^{N} \ln \left(1+\frac{|z|}{2 t^{2}}\right) d t\right) \leq \exp \left(\frac{(1+\pi) \sqrt{2}}{2} \sqrt{|z|}\right) .
\end{gathered}
$$

$-|z| \geq \frac{1}{\varepsilon}$. It follows that

$$
\prod_{n=1}^{N}\left(1+\frac{|z|+\varepsilon|z|^{2}}{4 n^{2}}\right) \leq \exp \left(\int_{0}^{N} \ln \left(1+\frac{\varepsilon|z|^{2}}{2 t^{2}}\right) d t\right) \leq \exp \left(\frac{(1+\pi) \sqrt{2 \varepsilon}}{2}|z|\right) .
$$

Consequently, we obtain that there exists a positive constant $C$ such that

$$
\left|E_{m}(z)\right| \leq C \exp \left(\frac{(1+\pi) \sqrt{2 \varepsilon}}{2}|z|\right) \quad(z \in \mathbb{C})
$$


Secondly, we have to evaluate $\left|Q_{m}\right|$.

$$
\begin{aligned}
\left|Q_{m}\right|= & \prod_{\substack{1 \leq|n| \leq N \\
n \neq m}}\left|\frac{\lambda_{n}}{\lambda_{n}-\lambda_{m}}\right|=\prod_{\substack{n=1 \\
n \neq m}}^{N}\left|\frac{\lambda_{n} \lambda_{-n}}{\lambda_{n} \lambda_{-n}-\left(\lambda_{-n}+\lambda_{n}\right) \lambda_{m}+\lambda_{m}^{2}}\right|=\prod_{\substack{n=1 \\
n \neq m}}^{N} \frac{\mu_{n}}{\left|\mu_{n}-\mu_{m}\right|} \leq \\
& \leq \prod_{\substack{n=1 \\
n \neq m}}^{N} \frac{n^{2}}{\left|n^{2}-m^{2}\right|}=\frac{\prod_{\substack{n=1 \\
n \neq m}}^{N} n^{2}}{\prod_{n=1}^{N-m} n \prod_{n=1}^{m-1} n \prod_{\substack{n=m+1 \\
n \neq 2 m}}^{N+m} n}=\frac{2 m}{m \prod_{n=N+1}^{N+m} n} \leq .
\end{aligned}
$$

Therefore, we have that

$$
\left|Q_{m}\right| \leq 2 \quad\left(m \in \mathbb{Z}^{*},|m| \leq N\right) .
$$

By using (5.3) and (5.4) we deduce that $P_{m}(z)$ is an entire function of exponential type at most $\frac{(1+\pi) \sqrt{2 \varepsilon}}{2}$ and the proof finishes.

The following result gives us the behavior of the product $P_{m}$ on the real axis.

Proposition 5.2. Given $h>0, \varepsilon>\frac{h^{2}}{16}$ and $m \in \mathbb{Z}^{*},|m| \leq N$, there exists a positive constant $\omega$, independent of $h, \varepsilon$ and $m$, such that

$$
\left|P_{m}(x)\right| \leq 2 \exp (\omega \varphi(x)) \quad(x \in \mathbb{R}),
$$

where $\varphi$ is given by

$$
\varphi(x)= \begin{cases}\sqrt{|x|} & |x|<\frac{1}{\varepsilon} \\ \sqrt{\varepsilon}|x| & \frac{1}{\varepsilon} \leq|x|<\frac{4}{h \sqrt{\varepsilon}} \\ \frac{\sqrt[4]{\varepsilon}}{\sqrt{h}} \sqrt{|x|} & \frac{4}{h \sqrt{\varepsilon}} \geq|x| .\end{cases}
$$

Proof. Since $\left|P_{m}(x)\right|=\left|E_{m}(x)\right|\left|Q_{m}\right|$ it follows from (5.4) that it is sufficient to evaluate $\left|E_{m}(x)\right|$. In the sequel, $C$ denotes a generic constant witch may change from one row to another but it is always independent of $h, \varepsilon$ and $m$. We begin the evaluation of $E_{m}(x)$.

$$
\left|E_{m}(x)\right|=\prod_{\substack{n=1 \\ n \neq m}}^{N}\left|1+\frac{i x}{\lambda_{n}}\right|\left|1+\frac{i x}{\lambda_{-n}}\right|=\underbrace{\prod_{\substack{n=1 \\ n \neq m}}^{n_{\varepsilon}}\left|1+\frac{i x}{\lambda_{n}}\right|\left|1+\frac{i x}{\lambda_{-n}}\right|}_{E_{m}^{1}(x)} \underbrace{\prod_{\substack{n=n_{\varepsilon}+1 \\ n \neq m}}^{N}\left|1+\frac{i x}{\lambda_{n}}\right|\left|1+\frac{i x}{\lambda_{-n}}\right|}_{E_{m}^{2}(x)} .
$$

By taking into account that both $E_{m}^{1}$ and $E_{m}^{2}$ are even functions of $x$, it is sufficient to consider only the case $x \geq 0$. Firstly, we estimate the product $E_{m}^{1}(x)$.

$$
E_{m}^{1}(x)=\prod_{\substack{n=1 \\ n \neq m}}^{n_{\varepsilon}}\left|1+\frac{i x}{\lambda_{n}}\right|\left|1+\frac{i x}{\lambda_{-n}}\right|=\left(1+\frac{x^{2}}{\lambda_{m}^{2}}\right)^{-\frac{1}{2}} \underbrace{\prod_{n=1}^{n_{\varepsilon}} \sqrt{1+\frac{x^{2}}{\lambda_{n}^{2}}}}_{E_{m}^{1+}(x)}\left(1+\frac{x^{2}}{\lambda_{-m}^{2}}\right)^{-\frac{1}{2}} \underbrace{\prod_{n=1}^{n_{\varepsilon}} \sqrt{1+\frac{x^{2}}{\lambda_{-n}^{2}}}}_{E_{m}^{1-}(x)} .
$$

Now, by taking into account (4.9) it follows that

$$
E_{m}^{1+}(x) \leq E_{m}^{1-}(x) \quad(x \geq 0),
$$


therefore in order to evaluate $E_{m}^{1}(x)$ it is enough to study $E_{m}^{1-}(x)$. By using, (4.8) and the inequality $\sin (t) \geq \frac{2}{\pi} t$, which holds for any $t \in\left(0, \frac{\pi}{2}\right)$, we deduce that

$$
\begin{gathered}
E_{m}^{1-}(x)=\prod_{n=1}^{n_{\varepsilon}} \sqrt{1+\frac{x^{2}}{\lambda_{-n}^{2}}} \leq \prod_{n=1}^{n_{\varepsilon}} \sqrt{1+\frac{x^{2}}{\mu_{n}^{2}}} \leq \\
\leq \exp \left(\frac{1}{2} \sum_{n=1}^{n_{\varepsilon}} \ln \left(1+\frac{x^{2}}{16 n^{4}}\right)\right) \leq \exp \left(\frac{1}{2} \int_{0}^{n_{\varepsilon}} \ln \left(1+\frac{x^{2}}{16 t^{4}}\right) d t\right) \leq \\
\leq \exp \left(\frac{1}{2} \int_{0}^{\frac{\sqrt{x}}{2}} \ln \left(1+\frac{x^{2}}{16 t^{4}}\right) d t+\frac{1}{2} \int_{\frac{\sqrt{x}}{2}}^{n_{\varepsilon}} \frac{x^{2}}{16 t^{4}} d t\right) \leq \exp (C \sqrt{x}) .
\end{gathered}
$$

Thus, we have that

$$
E_{m}^{1-}(x) \leq \exp (C \sqrt{x}) \quad(x \geq 0) .
$$

Consequently, from (5.7) and (5.8) we obtain that

$$
E_{m}^{1}(x) \leq \exp (C \sqrt{x}) \quad(x \geq 0) .
$$

Secondly, we evaluate $E_{m}^{2}(x)$ as follows

$$
\begin{aligned}
& \left|E_{m}^{2}(x)\right|^{2}=\left|\prod_{\substack{n=n_{\varepsilon}+1 \\
n \neq m}}^{N}\left(1+\frac{i x}{\lambda_{n}}\right)\left(1+\frac{i x}{\lambda_{-n}}\right)\right|^{2}=\prod_{\substack{n=n_{\varepsilon}+1 \\
n \neq m}}^{N}\left|\frac{\left|\lambda_{n}\right|^{2}+2 x i \Re\left(\lambda_{n}\right)-x^{2}}{\left|\lambda_{n}\right|^{2}}\right|^{2}= \\
& =\prod_{\substack{n=n_{\varepsilon}+1 \\
n \neq m}}^{N} \frac{\left(\left|\lambda_{n}\right|^{2}-x^{2}\right)^{2}+4 x^{2} \Re^{2}\left(\lambda_{n}\right)}{\left|\lambda_{n}\right|^{4}}=\prod_{\substack{n=n_{\varepsilon}+1 \\
n \neq m}}^{N}\left(1+\frac{x^{4}+4 x^{2} \Re^{2}\left(\lambda_{n}\right)-2 x^{2}\left|\lambda_{n}\right|^{2}}{\left|\lambda_{n}\right|^{4}}\right) .
\end{aligned}
$$

In order to complete the analysis of $E_{m}^{2}$ we have to treat separately the following two cases.

Case I. $x \leq \frac{1}{\varepsilon}$. By using (4.10) we obtain that

$$
\begin{aligned}
& \left|E_{m}^{2}(x)\right|^{2} \leq \prod_{\substack{n=n_{\varepsilon}+1 \\
n \neq m}}^{N}\left(1+\frac{8 x^{2} \Re^{2}\left(\lambda_{n}\right)-2 x^{2}\left|\lambda_{n}\right|^{2}}{\left|\lambda_{n}\right|^{4}}\right) \leq \\
& \leq \exp \left(\sum_{n=n_{\varepsilon}+1}^{N} \ln \left(1+\frac{8 x^{2} \Re^{2}\left(\lambda_{n}\right)}{\left|\lambda_{n}\right|^{4}}\right)\right) \leq \exp \left(\int_{n_{\varepsilon}}^{N} \ln \left(1+\frac{x^{2}}{8 t^{4}}\right) d t\right) \leq \\
& \exp \left(\int_{n_{\varepsilon}}^{\sqrt{x}} \ln \left(1+\frac{x^{2}}{8 t^{4}}\right) d t+\int_{\sqrt{x}}^{N} \frac{x^{2}}{8 t^{4}} d t\right) \leq \exp (C \sqrt{x})
\end{aligned}
$$

Case II. If $x>\frac{1}{\varepsilon}$ it results that

$$
\prod_{\substack{n=n_{\varepsilon}+1 \\ n \neq m}}^{N}\left(1+\frac{x^{4}+4 x^{2} \Re^{2}\left(\lambda_{n}\right)-2 x^{2}\left|\lambda_{n}\right|^{2}}{\left|\lambda_{n}\right|^{4}}\right) \leq \exp (\underbrace{\sum_{n=n_{\varepsilon}+1}^{N} \ln \left(1+\frac{x^{4}}{\left|\lambda_{n}\right|^{4}}\right)}_{S}) .
$$

To estimate $S$ we have to study the following cases. 
1. If $\frac{1}{\sqrt{\varepsilon}} \leq x \sqrt{\varepsilon} \leq \frac{4}{h}$ we have that

$$
\begin{aligned}
S & \leq \int_{\frac{1}{\sqrt{\varepsilon}}}^{\frac{4}{h}} \ln \left(1+\frac{\varepsilon^{2} x^{4}}{16 t^{4}}\right) d t=\int_{\frac{1}{\sqrt{\varepsilon}}}^{x \sqrt{\varepsilon}} \ln \left(1+\frac{\varepsilon^{2} x^{4}}{16 t^{4}}\right) d t+\int_{x \sqrt{\varepsilon}}^{\frac{4}{h}} \ln \left(1+\frac{\varepsilon^{2} x^{4}}{16 t^{4}}\right) d t= \\
& =\left.t \ln \left(1+\frac{\varepsilon^{2} x^{4}}{16 t^{4}}\right)\right|_{\frac{1}{\sqrt{\varepsilon}}} ^{x \sqrt{\varepsilon}}+4 \int_{\frac{1}{\sqrt{\varepsilon}}}^{x \sqrt{\varepsilon}} \frac{\varepsilon^{2} x^{4}}{16 t^{4}+\varepsilon^{2} x^{4}} d t+\int_{x \sqrt{\varepsilon}}^{\frac{4}{h}} \frac{\varepsilon^{2} x^{4}}{4 t^{4}} d t \leq C x \sqrt{\varepsilon} .
\end{aligned}
$$

2. For $x \sqrt{\varepsilon}>\frac{4}{h}$ it follows that

$$
S \leq \int_{\frac{1}{\sqrt{\varepsilon}}}^{\frac{4}{h}} \ln \left(1+\frac{\varepsilon^{2} x^{4}}{16 t^{4}}\right) d t=\left.t \ln \left(1+\frac{\varepsilon^{2} x^{4}}{16 t^{4}}\right)\right|_{\frac{1}{\sqrt{\varepsilon}}} ^{\frac{4}{h}}+\int_{\frac{1}{\sqrt{\varepsilon}}}^{\frac{4}{h}} \frac{4 \varepsilon^{2} x^{4}}{16 t^{4}+\varepsilon^{2} x^{4}} d t \leq C \frac{\sqrt[4]{\varepsilon} \sqrt{x}}{\sqrt{h}}
$$

where we have used the inequality $\ln (1+x) \leq 8 \sqrt[8]{x}$, which holds for any $x \in(1, \infty)$.

Therefore, for any $x \geq 0$ we obtain that

$$
\left|E_{m}^{2}(x)\right| \leq \begin{cases}\exp (C \sqrt{x}) & x \leq \frac{1}{\varepsilon} \\ \exp (C x \sqrt{\varepsilon}) & \frac{1}{\varepsilon} \leq x \leq \frac{4}{h \sqrt{\varepsilon}} \\ \exp \left(C \frac{\sqrt[4]{\varepsilon} \sqrt{x}}{\sqrt{h}}\right) & x>\frac{4}{h \sqrt{\varepsilon}} .\end{cases}
$$

Finally, from (5.9) and (5.10) we deduce that (5.5) takes place and the proof finishes.

Similarly, the following simpler result can be proved.

Proposition 5.3. Given $h>0, \varepsilon \leq \frac{h^{2}}{16}$ and $m \in \mathbb{Z}^{*},|m| \leq N$, there exists a positive constant $\omega$, independent of $h, \varepsilon$ and $m$, such that

$$
\left|P_{m}(x)\right| \leq 2 \exp (\omega \varphi(x)) \quad(x \in \mathbb{R}),
$$

where $\varphi$ is given by

$$
\varphi(x)=\sqrt{|x|} \quad(x \in \mathbb{R}) .
$$

Remark 5.4. Note that, in the case $\varepsilon \leq \frac{h^{2}}{16}$, the second branch of the function given by (5.6) disappears and the expression from the third one is bounded from above by $\sqrt{x}$. Hence, in this case, the function (5.6) is replaced by the simpler one given by (5.12). This result confirms the first part of Remark 4.3, in which we have said that, for $\varepsilon \leq \frac{h^{2}}{16}$, our problem is close to the continuous heat equation. Indeed, an estimate of the form (5.11) with $\varphi$ given by (5.12) is obtained for the continuous heat equation by Fattorini and Russell [5].

\subsection{A multiplier}

In this subsection we construct a multiplier $M_{m}$ which will compensate the grow on the real axis of the Weierstrass product $P_{m}$ given by (5.5).

We introduce the class $\Upsilon$ of continuous, increasing and onto functions $\varphi$ and we provide some technical results that we will be needed later on. Given $\varphi \in \Upsilon$ and $r \in \mathbb{N}^{*}$, we introduce the function

$$
\widetilde{\varphi}:[0, \infty) \rightarrow[0, \infty), \quad \widetilde{\varphi}(x)=\left\{\begin{array}{lr}
0 & \varphi(x)<r \\
\varphi(x)-r & \varphi(x) \geq r
\end{array}\right.
$$


and we define the sequences $\left(a_{n}\right)_{n \in \mathbb{N}^{*}}$ and $\left(n_{m}\right)_{\substack{m \in \mathbb{Z}^{*} \\|m| \leq N}}$ by

$$
\begin{gathered}
\varphi\left(e a_{n}\right)=n+r \quad\left(n \in \mathbb{N}^{*}\right), \\
n_{m}=\left\{\begin{array}{l}
1 \\
\varphi\left(e\left|\lambda_{m}\right|\right)<r \\
{\left[\varphi\left(e\left|\lambda_{m}\right|\right)\right]-r+1 \quad \varphi\left(e\left|\lambda_{m}\right|\right) \geq r}
\end{array} \quad\left(m \in \mathbb{Z}^{*},|m| \leq N\right) .\right.
\end{gathered}
$$

We have the following result.

Lemma 5.5. Let $x \geq a_{n_{m}}$ and define $n_{x}:=[\varphi(e x)]-r$. Then

$$
\sum_{j=n_{m}}^{n_{x}} \ln \left(\frac{a_{j}}{x}\right)=-\int_{a_{n_{m}}}^{x} \frac{A(u)-n_{m}+1}{u} d u
$$

where $A(u)=\#\left\{a_{n} \leq u\right\}=[\varphi(e u)]-r$.

Proof. We have that

$$
\begin{gathered}
-\int_{a_{n_{m}}}^{x} \frac{A(u)}{u} d u=-\sum_{j=n_{m}}^{n_{x}-1} \int_{a_{j}}^{a_{j+1}} \frac{A(u)}{u} d u-\int_{a_{n_{x}}}^{x} \frac{A(u)}{u} d u=-\sum_{j=n_{m}}^{n_{x}-1} \int_{a_{j}}^{a_{j+1}} \frac{j}{u} d u- \\
-\int_{a_{n_{x}}}^{x} \frac{n_{x}}{u} d u=\ln \left(\prod_{j=n_{m}}^{n_{x}-1} \frac{a_{j}^{j}}{a_{j+1}^{j}} \frac{a_{n_{x}}^{n_{x}}}{x^{n_{x}}}\right)=-\int_{a_{n_{m}}}^{x} \frac{n_{m}-1}{u} d u+\sum_{j=n_{m}}^{n_{x}} \ln \left(\frac{a_{j}}{x}\right),
\end{gathered}
$$

which completes the proof of the lemma.

Now, we can prove a general result which will give our desired multiplier function.

Proposition 5.6. Given $\varphi \in \Upsilon$ and $r \in \mathbb{N}^{*}$, let $\left(a_{n}\right)_{n \in \mathbb{N}^{*}}$ and $\left(n_{m}\right)_{\substack{m \in \mathbb{Z}^{*} \\|m| \leq N}}$ be given by (5.14) and (5.15), respectively. Suppose that, for each $m \in \mathbb{Z}^{*},|m| \leq N$, the following hypotheses hold:

$$
\begin{gathered}
\sum_{n \geq n_{m}} \frac{1}{a_{n}} \leq L, \\
\sum_{n \geq n_{m}} \frac{1}{a_{n}^{2}} \leq D \frac{1+\left|\Re\left(\lambda_{m}\right)\right|}{\left|\lambda_{m}\right|^{2}},
\end{gathered}
$$

where $L$ and $D$ are two positive constants independent of $m$. Then, for each $m \in \mathbb{Z}^{*}$ such that $|m| \leq N$, there exists $M_{m}: \mathbb{C} \rightarrow \mathbb{C}$ with the following properties:

1. $M_{m}$ is an entire function of exponential type at most $L$.

2. $\left|M_{m}(x)\right| \leq \exp \left(-\varphi(|x|)+\varphi\left(e\left|\lambda_{m}\right|\right)+r+1\right)$ for all $x \in \mathbb{R}$.

3. $\left|M_{m}\left(i \lambda_{m}\right)\right| \geq \exp \left(-\frac{D}{6}\left(1+\left|\Re\left(\lambda_{m}\right)\right|\right)\right)$.

Proof. By adapting an idea from [9], we define the function $M_{m}: \mathbb{C} \rightarrow \mathbb{C}$ as follows

$$
M_{m}(z)=\prod_{n=n_{m}}^{\infty} \frac{\sin \left(\frac{z}{a_{n}}\right)}{\frac{z}{a_{n}}},
$$

where the sequence $\left(a_{n}\right)_{n \geq 1}$ is given by (5.14) and $n_{m}$ by (5.15). 
$M_{m}$ is an entire function of exponential type. Indeed, this is a consequence of (5.16) and the following estimate

$$
\prod_{n=n_{m}}^{\infty}\left|\frac{\sin \left(\frac{z}{a_{n}}\right)}{\frac{z}{a_{n}}}\right| \leq \prod_{n=n_{m}}^{\infty} e^{\left|\frac{z}{a_{n}}\right|}=e^{|z| \sum_{n=n_{m}}^{\infty} \frac{1}{a_{n}}} \leq e^{L|z|} .
$$

To prove the second property of $M_{m}$ we have to treat separately the following two cases:

Case 1. $|x| \leq e a_{n_{m}}$. Taking into account that $\varphi \in \Upsilon$ it follows from the monotony of $\widetilde{\varphi}$, given by (5.13), that

$$
\widetilde{\varphi}(|x|) \leq \widetilde{\varphi}\left(e a_{n_{m}}\right) \leq \varphi\left(e\left|\lambda_{m}\right|\right)+1
$$

and consequently, we have that

$$
\begin{gathered}
\left|M_{m}(x)\right|=\prod_{n=n_{m}}^{\infty}\left|\frac{\sin \left(\frac{x}{a_{n}}\right)}{\frac{x}{a_{n}}}\right| \leq 1 \\
\leq \exp \left(-\widetilde{\varphi}(|x|)+\varphi\left(e\left|\lambda_{m}\right|\right)+1\right) \leq \exp \left(-\varphi(|x|)+\varphi\left(e\left|\lambda_{m}\right|\right)+r+1\right) .
\end{gathered}
$$

Case 2. $|x|>e a_{n_{m}}$. By using Lemma 5.5 we deduce that

$$
\left|M_{m}(x)\right| \leq \prod_{j=n_{m}}^{n_{x}}\left|\frac{a_{j}}{x}\right|=\exp \left(\sum_{j=n_{m}}^{n_{x}} \ln \left(\frac{a_{j}}{x}\right)\right) \leq \exp \left(-\int_{a_{n_{m}}}^{x} \frac{A(u)-n_{m}+1}{u} d u\right) .
$$

Since $a_{n_{m}}<\frac{x}{e}$ and $A(u)-n_{m}+1 \geq \varphi(e u)-\varphi\left(e\left|\lambda_{m}\right|\right)$, we obtain that

$$
\left|M_{m}(x)\right| \leq \exp \left(-\int_{\frac{x}{e}}^{x} \frac{A(u)-n_{m}+1}{u} d u\right) \leq \exp \left(-\varphi(|x|)+\varphi\left(e\left|\lambda_{m}\right|\right)+r+1\right) .
$$

Hence, the second property of $M_{m}$ is proved. Now, we pass to study the last property of $M_{m}$. We notice that

$$
a_{n_{m}}=\frac{1}{e} \varphi^{-1}\left(n_{m}+r\right) \geq \frac{1}{e} \varphi^{-1}\left(\varphi\left(e\left|\lambda_{m}\right|\right)-r+r\right)=\left|\lambda_{m}\right|,
$$

which implies that

$$
\frac{\left|\lambda_{m}\right|}{a_{n}} \leq 1 \quad\left(n \geq n_{m}\right)
$$

Thus, by taking into account (5.17) and the inequality

$$
\left|\frac{\sin x}{x}\right| \geq 1-\frac{1}{6} x^{2} \quad(|x| \leq 1)
$$

we deduce that

$$
\begin{aligned}
& \left|M_{m}\left(i \lambda_{m}\right)\right|=\prod_{n=n_{m}}^{\infty}\left|\frac{\sin \left(\frac{i \lambda_{m}}{a_{n}}\right)}{\frac{i \lambda_{m}}{a_{n}}}\right| \geq \prod_{n=n_{m}}^{\infty} \frac{\sin \left|\frac{\lambda_{m}}{a_{n}}\right|}{\left|\frac{\lambda_{m}}{a_{n}}\right|} \geq \prod_{n=n_{m}}^{\infty}\left|1-\frac{1}{6} \frac{\left|\lambda_{m}\right|^{2}}{a_{n}^{2}}\right|= \\
& =\exp \left(\sum_{n=n_{m}}^{\infty} \ln \left(1-\frac{1}{6} \frac{\left|\lambda_{m}\right|^{2}}{a_{n}^{2}}\right)\right) \geq \exp \left(-\frac{\left|\lambda_{m}\right|^{2}}{6} \sum_{n=n_{m}}^{\infty} \frac{1}{a_{n}^{2}}\right) \geq \exp \left(-\frac{D}{6}\left(1+\left|\Re\left(\lambda_{m}\right)\right|\right)\right) .
\end{aligned}
$$

This gives the third property of $M_{m}$ and completes the proof of the proposition.

In the following lemma we show that (5.16) and (5.17) are verified for the function $\varphi$ from (5.6). 
Lemma 5.7. Let $h, r>0, \varepsilon>\frac{h^{2}}{16}$ and $\varphi$ be given by (5.6). For every $m \in \mathbb{Z}^{*}$ such that $|m| \leq N$, the sequence $\left(a_{n}\right)_{n \in \mathbb{N}^{*}}$ defined by (5.14) has the following properties

$$
\begin{gathered}
\sum_{n \geq n_{m}} \frac{1}{a_{n}} \leq e\left(\frac{1}{r}+\sqrt{\varepsilon} \ln \left(\frac{4 \sqrt{\varepsilon}}{h}\right)+\frac{\sqrt{\varepsilon}}{4}\right), \\
\sum_{n \geq n_{m}} \frac{1}{a_{n}^{2}} \leq 33 \pi\left(\frac{1}{r}+4 h+\frac{\sqrt{\varepsilon}}{4}\right) \frac{1+\left|\Re\left(\lambda_{m}\right)\right|}{\left|\lambda_{m}\right|^{2}} .
\end{gathered}
$$

Proof. Since $\varphi$ is defined by (5.6), the sequence $\left(a_{n}\right)_{n \geq 1}$ will be given by

$$
a_{n}=\frac{1}{e} \begin{cases}(n+r)^{2} & n \leq\left[\frac{1}{\sqrt{\varepsilon}}\right]-r \\ \frac{n+r}{\sqrt{\varepsilon}} & {\left[\frac{1}{\sqrt{\varepsilon}}\right]^{-r} \leq n \leq\left[\frac{4}{h}\right]-r} \\ \frac{h(n+r)^{2}}{\sqrt{\varepsilon}} & {\left[\frac{4}{h}\right]-r \leq n .}\end{cases}
$$

To prove the first property we only have to remark that

$$
\begin{gathered}
\frac{1}{e} \sum_{n \geq n_{m}} \frac{1}{a_{n}} \leq \sum_{n=n_{m}}^{\left[\frac{1}{\sqrt{\varepsilon}}\right]-r}(n+r)^{-2}+\sum_{n=\left[\frac{1}{\sqrt{\varepsilon}}\right]-r+1}^{\left[\frac{4}{h}\right]-r} \frac{\sqrt{\varepsilon}}{n+r}+\sum_{n=\left[\frac{4}{h}\right]-r+1}^{\infty} \frac{\sqrt{\varepsilon}}{h}(n+r)^{-2} \leq \\
\leq \int_{0}^{\frac{1}{\sqrt{\varepsilon}}-r}(t+r)^{-2} d t+\int_{\frac{1}{\sqrt{\varepsilon}}-r}^{\frac{4}{h}-r} \frac{\sqrt{\varepsilon}}{t+r} d t+\frac{\sqrt{\varepsilon}}{h} \int_{\frac{4}{h}-r}^{\infty}(t+r)^{-2} d t \leq \frac{1}{r}+\sqrt{\varepsilon} \ln \left(\frac{4 \sqrt{\varepsilon}}{h}\right)+\frac{\sqrt{\varepsilon}}{4},
\end{gathered}
$$

which gives (5.19).

Now, we pass to study property (5.20). Firstly, we have that

$$
\begin{gathered}
\frac{1}{e^{2}} \sum_{n \geq n_{m}} \frac{1}{a_{n}^{2}}=\sum_{n=n_{m}}^{\left[\frac{1}{\sqrt{\varepsilon}}\right]-r} \frac{1}{(n+r)^{4}}+\sum_{n=\left[\frac{1}{\sqrt{\varepsilon}}\right]-r+1}^{\left[\frac{4}{h}\right]-r} \frac{\varepsilon}{(n+r)^{2}}+\sum_{n=\left[\frac{4}{h}\right]-r+1}^{\infty} \frac{\varepsilon}{h^{2}} \frac{1}{(n+r)^{4}} \leq \\
\leq \int_{n_{m}-1}^{\frac{1}{\sqrt{\varepsilon}}-r}(t+r)^{-4} d t+\varepsilon \int_{\frac{1}{\sqrt{\varepsilon}}-r}^{\frac{4}{h}-r}(t+r)^{-2} d t+\frac{\varepsilon}{h^{2}} \int_{\frac{4}{h}-r}^{\infty}(t+r)^{-4} d t \leq \\
\frac{1}{3}\left(\frac{1}{\left(n_{m}-1+r\right)^{3}}+\frac{193 \varepsilon h}{64}\right) .
\end{gathered}
$$

Since $\frac{1+\left|\Re\left(\lambda_{m}\right)\right|}{\left|\lambda_{m}\right|^{2}} \geq \frac{1}{\left|\lambda_{m}\right|}$ and $\varepsilon h\left|\lambda_{m}\right| \leq \pi \sqrt{\varepsilon}+h$, inequality (5.20) takes place if

$$
\frac{\left|\lambda_{m}\right|}{\left(n_{m}-1+r\right)^{3}} \leq \frac{2 \pi}{e^{2}}\left(\frac{1}{r}+\sqrt{\varepsilon}\right) .
$$

To show (5.22), we analyze separately the following two cases.

Case 1: If $\varphi\left(e\left|\lambda_{m}\right|\right)<r$ it follows from (5.15) that $n_{m}=1$. In this case from the definition of the function $\varphi$, properties (4.8) and (4.8) we deduce that $\mu_{|m|}<\frac{r^{2}}{e}$ and

$$
\frac{\left|\lambda_{m}\right|}{\left(n_{m}-1+r\right)^{3}}=\frac{\left|\lambda_{m}\right|}{r^{3}} \leq \frac{2 \mu_{|m|}}{r^{3}} \leq \frac{2}{e r},
$$

which gives (5.22). 
Case 2: If $\varphi\left(e\left|\lambda_{m}\right|\right) \geq r$, by using again the definition of $\varphi$, relation (4.10) and (4.7) we have that

$$
\frac{\left|\lambda_{m}\right|}{\left(n_{m}-1+r\right)^{3}}=\frac{\left|\lambda_{m}\right|}{\left(\varphi\left(e\left|\lambda_{m}\right|\right)\right)^{3}} \leq \frac{2 \pi}{e^{2}} \frac{1}{r}+\frac{\sqrt{\varepsilon}}{e^{3}}
$$

which gives again (5.22).

This completes the proof of the lemma.

Similarly, the following simpler result can be proved.

Lemma 5.8. Let $h, r>0, \varepsilon \leq \frac{h^{2}}{16}$ and $\varphi$ be given by (5.12). For every $m \in \mathbb{Z}^{*}$ such that $|m| \leq N$, the sequence $\left(a_{n}\right)_{n \in \mathbb{N}^{*}}$ defined by (5.14) has the following properties

$$
\begin{gathered}
\sum_{n \geq n_{m}} \frac{1}{a_{n}} \leq \frac{e}{r} \\
\sum_{n \geq n_{m}} \frac{1}{a_{n}^{2}} \leq \frac{e}{3 r} \frac{1+\left|\Re\left(\lambda_{m}\right)\right|}{\left|\lambda_{m}\right|^{2}} .
\end{gathered}
$$

Now, we have all the ingredients needed to construct the desired multiplier function. This is done in the following proposition.

Proposition 5.9. Let $T>0$. There exist $h_{0}, c_{0} \in(0,1)$ and $r_{0}>0$ such that for each $h \in\left(0, h_{0}\right)$, $\varepsilon \in\left(0, c_{0} h\right), r>r_{0}$ and $m \in \mathbb{Z}^{*}$ such that $|m| \leq N$, there exists a function $M_{m}: \mathbb{C} \rightarrow \mathbb{C}$ with the following properties:

1. $M_{m}$ is an entire function of exponential type at most $\frac{T}{32 \omega}$.

2. $\left|M_{m}(x)\right| \leq C \exp \left(-\varphi(|x|)+\frac{T}{32 \omega}\left|\Re\left(\lambda_{m}\right)\right|\right)$ for all $x \in \mathbb{R}$.

3. $\left|M_{m}\left(i \lambda_{m}\right)\right| \geq \exp \left(-\frac{T}{32 \omega}\left(\left|\Re\left(\lambda_{m}\right)\right|+1\right)\right)$,

where $\omega$ is the constant from (5.5), the function $\varphi$ is given by (5.6) or (5.12) and $C$ is a positive constant independent of $h, \varepsilon$ and $m$.

Proof. Firstly, let us remark that there exist $h_{0}, c_{0} \in(0,1)$ and $r_{0}^{\prime}>0$ such that

$$
\begin{gathered}
e\left(\frac{1}{r}+\sqrt{\varepsilon} \ln \left(\frac{4 \sqrt{\varepsilon}}{h}\right)+\frac{\sqrt{\varepsilon}}{4}\right)<\frac{T}{32 \omega} \quad\left(h \in\left(0, h_{0}\right), \varepsilon \in\left(0, c_{0} h\right), r>r_{0}^{\prime}\right), \\
33 \pi\left(\frac{1}{r}+4 h+\frac{\sqrt{\varepsilon}}{4}\right)<\frac{T}{32 \omega} \quad\left(h \in\left(0, h_{0}\right), \varepsilon \in\left(0, c_{0} h\right), r>r_{0}^{\prime}\right), \\
e^{2} \pi c_{0} \leq \frac{T}{32 \omega} .
\end{gathered}
$$

By taking into account Proposition 5.6, Lemmas 5.7 and 5.8, relations (5.25) and (5.26) it follows immediately that properties 1 and 3 of $M_{m}$ are verified. In order to obtain the second property of $M_{m}$, it is sufficient to prove that, there exists $r_{0}>r_{0}^{\prime}$ such that the following inequality holds for $r \geq r_{0}$

$$
\varphi\left(e\left|\lambda_{m}\right|\right) \leq \frac{T}{32 \omega}\left|\Re\left(\lambda_{m}\right)\right|+r .
$$

To show (5.28) we note that, if $\varepsilon \leq \frac{h^{2}}{16}$, the following inequality holds

$$
\sqrt{e\left|\lambda_{m}\right|} \leq \frac{T}{32 \omega}\left|\lambda_{m}\right|+r,
$$


which gives (5.28) for $r$ sufficiently large. The same argument can be used to obtain (5.28) in the case $\varepsilon>\frac{h^{2}}{16}$ and $e\left|\lambda_{m}\right|<\frac{1}{\varepsilon}$.

Finally, if $\varepsilon>\frac{h^{2}}{16}$ and $\frac{1}{\varepsilon} \leq e\left|\lambda_{m}\right| \leq \frac{4}{h \sqrt{\varepsilon}}$, then we have that

$$
\sqrt{\varepsilon} e\left|\lambda_{m}\right| \frac{1}{\Re\left(\lambda_{m}\right)} \leq e^{2} \varepsilon \sqrt{\varepsilon}\left|\lambda_{m}\right| \leq e^{2} \pi c_{0}<\frac{T}{32 \omega}
$$

which completes the proof of (5.28).

Consequently the proof of the second property of $M_{m}$ and of the entire proposition ends.

\subsection{The biorthogonal sequence}

Now, we have all the ingredients needed to show the existence of a biorthogonal sequence to the family of exponential functions $\left(e^{\lambda_{n} t}\right)_{\substack{|n| \leq N \\ n \neq 0}}$ in $L^{2}(0, T)$.

Theorem 5.10. Let $T>0$. There exist $h_{0}, c_{0} \in(0,1)$ such that for any $h \in\left(0, h_{0}\right)$ and $\varepsilon \in\left(0, c_{0} h\right)$ there

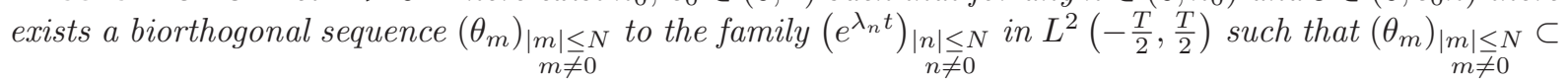
$\mathcal{C}^{1}\left[-\frac{T}{2}, \frac{T}{2}\right]$ and

$$
\left\|\theta_{m}\right\|_{\mathcal{C}^{1}\left[-\frac{T}{2}, \frac{T}{2}\right]} \leq C \exp \left(\alpha\left|\Re\left(\lambda_{m}\right)\right|\right) \quad\left(m \in \mathbb{Z}^{*},|m| \leq N\right),
$$

where $\alpha<\frac{T}{4}$ and $C$ is a positive constant independent of $h, \varepsilon$ and $m$.

Proof. For each $m \in \mathbb{Z}^{*}$, such that $|m| \leq N$, let $P_{m}$ and $M_{m}$ be the functions from Propositions 5.1 and 5.9 , respectively. We define the function

$$
\Psi_{m}(z)=P_{m}(z)\left(\frac{M_{m}(z)}{M_{m}\left(i \lambda_{m}\right)}\right)^{2 \omega} \frac{\sin \left(\delta\left(z-i \lambda_{m}\right)\right)}{\delta\left(z-i \lambda_{m}\right)}
$$

where $\delta \in\left(0, \frac{T}{8}\right)$ is an arbitrary constant and $\omega$ is the same as in Proposition $5.2\left(\right.$ if $\left.\varepsilon>\frac{h^{2}}{16}\right)$ or Proposition 5.3 (if $\left.\varepsilon \leq \frac{h^{2}}{16}\right)$. Let

$$
\theta_{m}(t)=\frac{1}{2 \pi} \overline{\int_{\mathbb{R}} \Psi_{m}(x) e^{i x t} d x} \quad(|m| \leq N) .
$$

From Propositions 5.1 and 5.9 and formula (5.31) we deduce that $\Psi_{m}$ is an entire function of exponential type at most $\frac{(1+\pi) \sqrt{2 \varepsilon}}{2}+\frac{T}{16}+\delta$. Since $\delta<\frac{T}{8}$ it follows that there exist $h_{0}, c_{0} \in(0,1)$ such that for any $h \in\left(0, h_{0}\right), \varepsilon \in\left(0, c_{0} h\right)$ we have that $\Psi_{m}$ is an entire function of exponential type at most $\frac{T}{2}$.

By using Paley-Wiener Theorem [27, Theorem 18, Sec. 2.4] and the decay properties of $\Psi_{m}$ on the real axis we deduce that $\theta_{m} \in \mathcal{C}^{1}\left[-\frac{T}{2}, \frac{T}{2}\right]$ ( in fact $\theta_{m} \in \mathcal{C}^{k}\left[-\frac{T}{2}, \frac{T}{2}\right]$, for any $k \in \mathbb{N}^{*}$ ). From the Inverse Fourier Theorem and (5.2), we deduce that $\left(\theta_{m}\right)_{\substack{|m| \leq N \\ m \neq 0}}$ is biorthogonal to the family $\left(e^{\lambda_{n} t}\right)_{\substack{|n| \leq N \\ n \neq 0}}$ in $L^{2}\left(-\frac{T}{2}, \frac{T}{2}\right)$. Moreover, from the estimate of the function $P_{m}$ on the real axis given by Propositions 5.2 and 5.3, the properties of the function $M_{m}$ from Proposition 5.9 and taking into account that

$$
\left|\frac{\sin z}{z}\right| \leq \frac{e^{\Im(z)}}{\Im(z)} \quad(z \in \mathbb{C}),
$$


we obtain that the following estimates hold

$$
\begin{aligned}
& \left\|\theta_{m}(t)\right\|_{L^{\infty}\left(-\frac{T}{2}, \frac{T}{2}\right)}=\left|\frac{1}{2 \pi} \int_{\mathbb{R}} \Psi_{m}(x) e^{i x t} d x\right| \leq \\
& \leq C \exp \left(\frac{T}{8}\left|\Re\left(\lambda_{m}\right)\right|\right) \int_{\mathbb{R}}\left|\frac{\sin \left(\delta\left(x-i \lambda_{m}\right)\right)}{\delta\left(x-i \lambda_{m}\right)}\right| e^{-\omega \varphi(x)} d x \\
& \leq \frac{C}{\delta} \exp \left(\left(\frac{T}{8}+\delta\right)\left|\Re\left(\lambda_{m}\right)\right|\right) \int_{\mathbb{R}} e^{-\omega \sqrt{x}} d x \leq C \exp \left(\alpha\left|\Re\left(\lambda_{m}\right)\right|\right),
\end{aligned}
$$

where $\alpha=\frac{T}{8}+\delta<\frac{T}{4}$ and $C$ is a positive constant independent of $h, \varepsilon$ and $m$.

Moreover, similar to (5.32), the following estimates take place

$$
\left\|\theta_{m}^{\prime}(t)\right\|_{L^{\infty}\left(-\frac{T}{2}, \frac{T}{2}\right)}=\left|\frac{1}{2 \pi} \int_{\mathbb{R}} i x \Psi_{m}(x) e^{i x t} d x\right| \leq C \exp \left(\alpha\left|\Re\left(\lambda_{m}\right)\right|\right) .
$$

From (5.32) and (5.33) it results that (5.29) holds and the proof of the theorem finishes.

\section{Controllability results}

The aim of this section is to use the biorthogonal sequence from Theorem 5.10 in order to construct a uniformly bounded sequence of controls for the discrete perturbed equation (3.4) with the property that any weak limit of it is a control for the continuous problem (2.1). We have the following result concerning the uniform boundeness of the family of discrete controls.

Theorem 6.1. Let $T>0$. There exist $h_{0}, c_{0} \in(0,1)$ such that for any $h \in\left(0, h_{0}\right), \varepsilon \in\left(0, c_{0} h\right)$ and any initial data $\left(U_{h}^{0}, U_{h}^{1}\right) \in \mathbb{C}^{2 N}$ of the form

$$
\left(U_{h}^{0}, U_{h}^{1}\right)=\left(\sum_{j=1}^{N} a_{j h}^{0} \varphi^{j}, \sum_{j=1}^{N} a_{j h}^{1} \varphi^{j}\right)
$$

such that

$$
\sum_{\substack{|n| \leq N \\ n \neq 0}} e^{(-T+4 \alpha)\left|\Re\left(\lambda_{n}\right)\right|}\left(\left|a_{|n| h}^{1}\right|^{2}+\left|a_{|n| h}^{0}\right|^{2}\right)<\infty
$$

there exists a control $v_{h} \in \mathcal{C}^{1}[0, T]$ of the discrete perturbed wave equation (3.6) with the property that the family $\left(v_{h}\right)_{h}$ is uniformly bounded in $\mathcal{C}^{1}[0, T]$.

Proof. For any initial data $\left(U_{h}^{0}, U_{h}^{1}\right) \in \mathbb{C}^{2 N}$ we construct the function $v_{h} \in \mathcal{C}^{1}[0, T]$ as follows

$$
v_{h}(t)=\sum_{\substack{|n| \leq N \\ n \neq 0}} \frac{(-1)^{n} h e^{-\bar{\lambda}_{n} \frac{T}{2}}}{\sqrt{2} \sin (|n| \pi h)}\left(\varepsilon a_{|n| h}^{1}-\varepsilon a_{|n| h}^{0} \bar{\lambda}_{n}+a_{|n| h}^{0}\right) \theta_{n}\left(t-\frac{T}{2}\right) \quad(t \in(0, T)),
$$

where $\left(\theta_{m}\right)_{\substack{|m| \leq N \\ m \neq 0}}$ is the biorthogonal sequence to the family $\left(e^{\lambda_{n} t}\right)_{\substack{|n| \leq N \\ n \neq 0}}$ given by Theorem 5.10. Let us show that $v_{h}$ is the control we are looking for. In results from Theorem 5.10 that $v_{h} \in \mathcal{C}^{1}[0, T]$. From the properties of the biorthogonal sequence $\left(\theta_{n}\right)_{n}$, it is easy to see that $v_{h}$ verifies (4.13). To conclude that 
$v_{h}$ is a control for (3.6), we only have to prove that the right hand side of $(6.3)$ is bounded in $\mathcal{C}^{1}[0, T]$. This follows immediately from Theorem 5.10. Indeed, we have that

$$
\begin{gathered}
\left\|v_{h}\right\|_{\mathcal{C}^{1}[0, T]} \leq C \sum_{\substack{|n| \leq N \\
n \neq 0}}\left|\frac{h e^{-\frac{T}{2}\left|\Re\left(\lambda_{n}\right)\right|}}{\sqrt{2} \sin (n \pi h)}\right|\left|\varepsilon a_{|n| h}^{1}-\varepsilon a_{|n| h}^{0} \bar{\lambda}_{n}+a_{|n| h}^{0}\right|\left(\left\|\theta_{n}\right\|_{L^{\infty}}+\left\|\theta_{n}^{\prime}\right\|_{L^{\infty}}\right) \leq \\
\leq C \sum_{\substack{|n| \leq N \\
n \neq 0}} \frac{h}{\sqrt{2}|\sin (n \pi h)|} e^{\left(-\frac{T}{2}+\alpha\right)\left|\Re\left(\lambda_{n}\right)\right|}\left(\varepsilon\left|a_{|n| h \mid}^{1}\right|+\varepsilon\left|\lambda_{n}\right|\left|a_{|n| h \mid}^{0}\right|+\left|a_{|n| h}^{0}\right|\right) \leq \\
\leq C\left[\sum_{\substack{|n| \leq N \\
n \neq 0}} e^{(-T+4 \alpha)\left|\Re\left(\lambda_{n}\right)\right|}\left(\left|a_{|n| h}^{1}\right|^{2}+\left|a_{|n| h}^{0}\right|^{2}\right)\right]^{\frac{1}{2}}\left[\sum_{\substack{|n| \leq N \\
n \neq 0}}\left|\frac{h^{2} e^{-2 \alpha\left|\Re\left(\lambda_{n}\right)\right|}}{2 \sin ^{2}(n \pi h)}\right|\right]^{\frac{1}{2}} \leq C,
\end{gathered}
$$

where $C$ is a positive constant independent of $h, \varepsilon$ and $m$.

The main result of this paper reads as follows.

Theorem 6.2. Let $T>0$ and $u^{0} \in L^{2}(0,1)$ given by (2.4). There exist $h_{0}, c_{0} \in(0,1)$ such that for any $h \in\left(0, h_{0}\right), \varepsilon \in\left(0, c_{0} h\right)$ and any initial data $\left(U_{h}^{0}, U_{h}^{1}\right) \in \mathbb{C}^{2 N}$ of the form

$$
\left(U_{h}^{0}, U_{h}^{1}\right)=\left(\sum_{j=1}^{N} a_{j h}^{0} \varphi^{j}, 0\right)
$$

and with the property that

$$
\left(a_{n h}^{0}\right)_{n} \rightarrow\left(a_{n}\right)_{n} \text { in } \ell^{2} \text { when } h \rightarrow 0,
$$

there exists a family of exact controls $\left(v_{h}\right)_{h>0} \subset \mathcal{C}^{1}[0, T]$ for problem (3.4) which converges to a nullcontrol for (2.1) in $\mathcal{C}[0, T]$ when $h$ tends to zero.

Proof. Firstly, we note that (6.5) implies that (6.2) is verified. Now, we consider the family $\left(v_{h}\right)_{h}$ defined in Theorem 6.1 which is uniformly bounded in $\mathcal{C}^{1}[0, T]$. By using Arzela-Ascoli Theorem, it follows that there exists a subfamily of $\left(v_{h}\right)_{h}$, denoted in the same way, which converges to a function $v$ in $\mathcal{C}[0, T]$.

Since $v_{h}$ is a control for (3.4) it follows from Theorem 4.5 that

$$
\int_{0}^{T} v_{h}(t) e^{\bar{\lambda}_{n} t} d t=\frac{(-1)^{n+1} h}{\sqrt{2} \sin (|n| \pi h)}\left(-\varepsilon a_{|n| h}^{1}+\varepsilon \bar{\lambda}_{n} a_{|n| h}^{0}-a_{|n| h}^{0}\right) \quad\left(-1 \leq n \leq-\frac{1}{\sqrt[4]{\varepsilon}}\right) .
$$

Now, by passing to the limit as $h \rightarrow 0$ in (6.6) and taking into account that $\varepsilon \rightarrow 0$ too, we deduce that

$$
(-1)^{n+1} \int_{0}^{T} v(t) e^{n^{2} \pi^{2} t} d t=-\frac{1}{\sqrt{2} n \pi} a_{n} \quad\left(n \in \mathbb{N}^{*}\right) .
$$

Finally, from (2.5) we obtain that $v$ is a null-control for (2.1) and the proof of the theorem ends.

Remark 6.3. The usual discretization by points

$$
\left(U_{h}^{0}, U_{h}^{1}\right)=\left(\left(u^{0}(j h)\right)_{1 \leq j \leq N},\left(u^{1}(j h)\right)_{1 \leq j \leq N}\right),
$$

leads to a convergence property of the Fourier coefficients sequence that depends on the regularity of $\left(u^{0}, u^{1}\right)$. Indeed, it is not difficult to prove the following: 
(i) If $u^{0}$ and $u^{1}$ are piecewise continuous functions in $[0,1]$ then

$$
\left(a_{n h}^{0}\right)_{n} \rightarrow\left(a_{n}^{0}\right)_{n} \quad\left(\frac{a_{n h}^{1}}{\lambda_{n}}\right)_{n} \rightarrow\left(\frac{a_{n}^{1}}{n \pi i}\right) \text { when } h \rightarrow 0 \text { in } l^{1} .
$$

(ii) If $u^{0}$ and $u^{1}$ are one time derivable with continuous derivative in $[0,1]$ then

$$
\left(a_{n h}^{0}\right)_{n} \rightarrow\left(a_{n}^{0}\right)_{n} \quad\left(\frac{a_{n h}^{1}}{\lambda_{n}}\right)_{n} \rightarrow\left(\frac{a_{n}^{1}}{n \pi i}\right) \text { when } h \rightarrow 0 \text { in } l^{1} .
$$

\section{Numerical results}
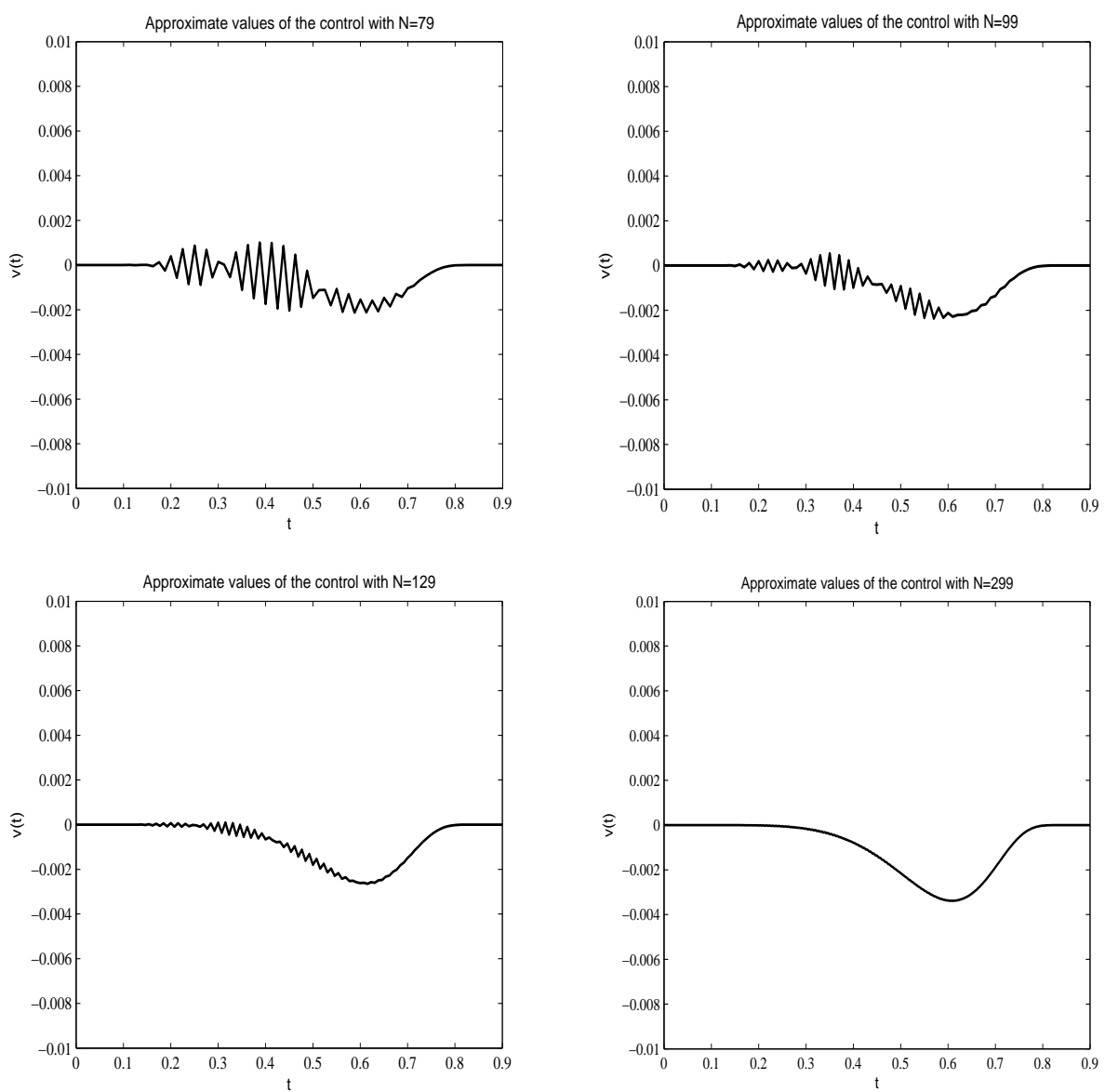

Figure 2. Numerical example 1 - Approximations of the control $\widehat{v}_{h}$ for $\mathrm{N}=79,99,129,299$.

In this section we present two numerical experiments to approximate the control of (2.1) based on discretization (3.4), with the singular perturbation, $\varepsilon u^{\prime \prime}$. More precisely, we approximate the HUM control $v$ for (2.1) (the control of minimal $L^{2}$-norm with respect to some weight if required, see $[4,19]$ ) by using (3.4) as discretization of (2.1).

The algorithm we have used to compute the approximate controls is based on a conjugate gradient implementation of the HUM method inspired in [4]. To this end, by considering approximation $U_{h}^{0}$ of $u^{0}$, 


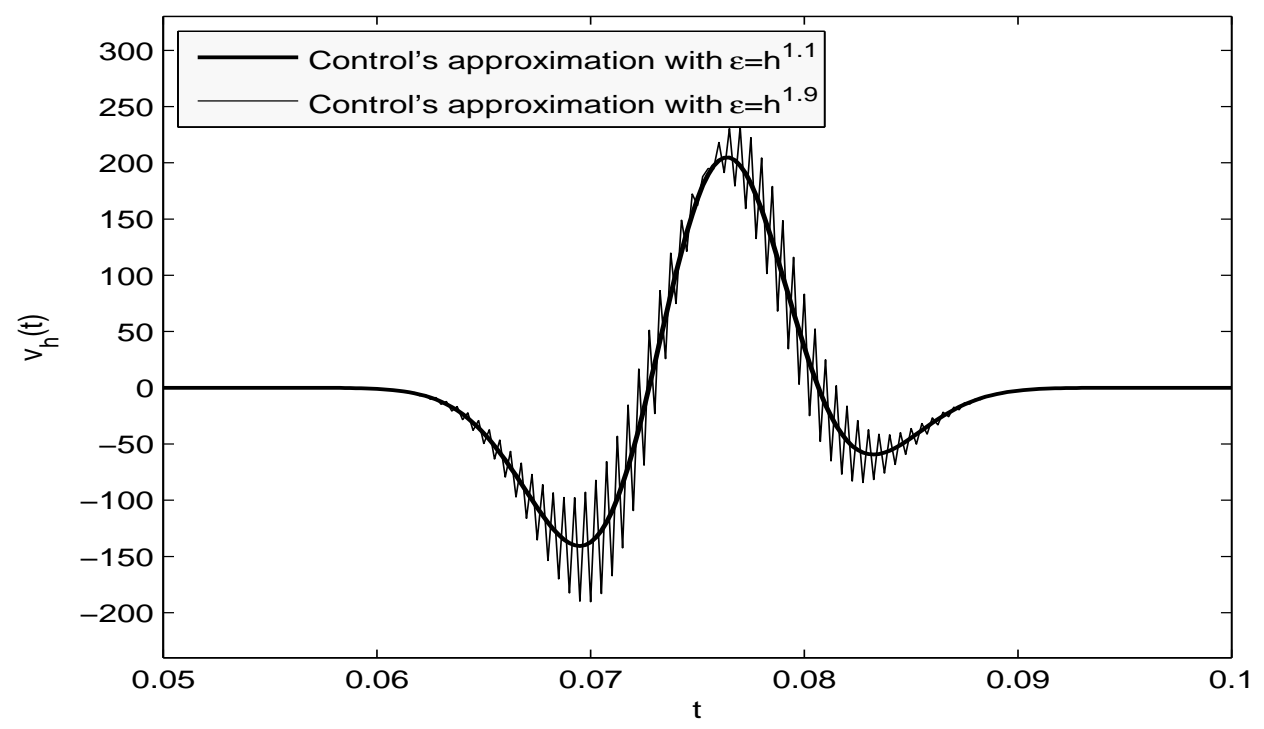

Figure 3. Numerical example 2 - Approximations of the control $\widehat{v}_{h}$ for two different values of $\varepsilon$.

we minimize the functional $J: \mathbb{C}^{2 N} \rightarrow \mathbb{C}$ defined by

$$
J\left(W_{h}^{0}, W_{h}^{1}\right)=\frac{1}{2} \int_{0}^{T} \vartheta(t)\left|\frac{W_{h, N}(t)}{h}\right|^{2} d t+\left\langle U_{h}^{0}, W_{h}(0)\right\rangle-\varepsilon\left\langle U_{h}^{0}, W_{h}^{\prime}(0)\right\rangle,
$$

where $\left(W_{h}, W_{h}^{\prime}\right)$ is the solution of (4.1) with the initial data $\left(W_{h}^{0}, W_{h}^{1}\right)$. In (7.1) $\vartheta \in C^{\infty}[0, T]$ is a cut-off regular function with the property that there exists a positive number $\epsilon<\frac{T}{2}$ such that

$$
\operatorname{supp}(\vartheta) \subset(\epsilon / 2, T-\epsilon / 2), \quad 0 \leq \vartheta(t) \leq 1 \text { for all } t \in[0, T], \quad \vartheta(t) \geq 1 / 2 \text { for all } t \in[\epsilon, T-\epsilon] .
$$

The function $\vartheta$ has the role to improve the numerical approximations of the controls, avoiding incompatibility between the initial data and the nonhomogeneous term at the origin.

The minimizer $\left(\widehat{W}_{h}^{0}, \widehat{W}_{h}^{1}\right)$ of $J$ provides the control $\widehat{v}_{h}$ of the discrete system (3.4) with minimal $L^{2}$-norm with respect to the weight $\vartheta$. The weak limit of the family $\left(\widehat{v}_{h}\right)_{h>0}$ is the control of $(2.1)$ mention before.

In the algorithm several wave equations have to be solved. In order to solve the differential equations in $t$ we use the Newmark Method with the parameters $\gamma=0.5$ and $\beta=0.25$ (see [8]).

Numerical example 1: In this example we take $T=0.9, \varepsilon=h^{1.1}$ and the initial data to be controlled

$$
u^{0}(x)=10 x(1-x) \quad(x \in(0,1)) .
$$

The approximations of the control, for different values of $N$, are presented in Figure 2 in which the convergence of the controls $\widehat{v}_{h}$ when $h$ and $\varepsilon$ go to zero can be immediately observed.

Numerical example 2: In this case we take $T=0.15, N=3999$ and the initial data

$$
u^{0}(x)=\sin (\pi x) \quad(x \in(0, \pi)) .
$$

The approximation of the control, for two different values of $\varepsilon$, are presented in Figure 3.

Note that, at least in this case, the introduction of the perturbation $\varepsilon u^{\prime \prime}$ has improved the numerical results in the approximations $\widehat{v}_{h}$. Indeed, if $\varepsilon=h^{1.9}$ the perturbation is very small and we are close to the 
case of the unperturbed situation (3.1). If $\varepsilon=h^{1.1}$ the perturbation has a stronger influence, regularizes the controls and produces better numerical results.

Acknowledgements. The first author was partially supported by the strategic grant POSDRU/CPP107/ DMI1.5/S/78421, Project ID 78421 (2010), co-financed by the European Social Fund - Investing in People, within the Sectorial Operational Programme Human Resources Development 2007-2013. The second author was partially supported by Grant PN-II-ID-PCE-2011-3-0257 of the Romanian National Authority for Scientific Research, CNCS UEFISCDI and by Grant MTM2011-29306 funded by MICINN (Spain).

\section{References}

[1] S.A. Avdonin, S.A. Ivanov. Families of exponentials. The method of moments in controllability problems for distributed parameter systems. Cambridge University Press, 1995.

[2] F. Boyer, J. Le Rousseau. Carleman estimates for semi-discrete parabolic operators and application to the controllability of semi-linear semi-discrete parabolic equations. Ann. Inst. Poincarè, Available online 12 September 2013 , http://dx.doi.org/10.1016/j.anihpc.2013.07.011.

[3] E. Fernàndez-Cara, A. Münch. Numerical null-controllability of a semi-linear heat equation via a least squares method. C. R. Acad. Sci. Paris, Ser. I 340 (2005).

[4] C. Carthel, R. Glowinski, J.-L. Lions. On exact and approximate Boundary Controllability for the heat equation: A numerical approach. JOTA 82 (1994), 429-484.

[5] H.O. Fattorini, D.L. Russell. Exact controllability theorems for linear parabolic equations in one space dimension. Arch. Ration. Mech. Anal., 43 (1971), 272-292.

[6] H.O. Fattorini, D.L. Russell. Uniform bounds on biorthogonal functions for real exponentials with an application to the control theory of parabolic equations. Q. Appl. Math., 32 (1974/75), 45-69.

[7] A.V. Fursikov, O.Yu. Imanuvilov. Controllability of Evolution Equations. Lecture Notes Series, Number 34, Seoul National University, Korea, 1996.

[8] T.J.R. Hughes. The finite element method: Linear static and dynamic finite element analysis. Prentice Hall Inc., Englewood Cliffs, NJ, 1987.

[9] A.E. Ingham. A note on Fourier transform. J. London Math. Soc., 9 (1934), 29-32.

[10] E. Isaakson, H.B. Keller. Analysis of Numerical Methods, John Wiley and Sons, 1996.

[11] V. Komornik, P. Loreti. Fourier Series in Control Theory, Springer-Verlag, New-York, 2005.

[12] R. Lattés, J.-L. Lions. The Method of Quasi-Reversibility. Applications to Partial Differential Equations. Modern Analytic and Computational Methods in Science and Mathematics vol 18, New York: American Elsevier, 1969.

[13] G. Lebeau, L. Robbiano. Contrôle exact de l'équation de la chaleur. Comm. Partial Differential Equations 20 (1995), $335-356$.

[14] J. L. Lions. Controlabilité exacte, stabilisation et perturbations des systèmes distribués. Vol. 1, Masson, Paris, 1988.

[15] J.-L. Lions, E. Zuazua. The cost of controlling unstable systems: time irreversible systems. Rev. Mat. de la UCM 10 (1997), 481-523.

[16] J.-L. Lions, E. Zuazua. The cost of controlling unstable systems: the case of boundary controls, J. Anal. Math. 73 (1997), 225-249.

[17] A. López, E. Zuazua. Some new results related to the null-controllability of the 1-d heat equation. Sèm EDP, Ecole Polytech. VIII (1998), 1-22.

[18] A. López, X. Zhang, E. Zuazua. Null controllability of the heat equation as singular limit of the exact controllability of dissipative wave equations. J. Math. Pures Appl. 79 (2000), 741-808.

[19] S. Micu, E. Zuazua. An Introduction to the Controllability of Partial Differential Equations. "Quelques questions de thèorie du contròle". Sari, T., ed., Collection Travaux en Cours Hermann, (2004), 69-157.

[20] S. Micu, E. Zuazua. On the regularity of null-controls of the linear 1-d heat equation. C. R. Acad. Sci. Paris, Ser. I 349 (2011), 673-677.

[21] S. Micu, E. Zuazua. Regularity issues for the null-controllability of the linear 1-d heat equation. Systems \& Control Letters 60 (2011), 406-413.

[22] A. Münch, P. Pedregal. Numerical null controllability of the heat equation through a least squares and variational approach. European Journal of Applied Mathematics, Published online: 13 February 2014, http://dx.doi.org/10.1017/S0956792514000023.

[23] A. Münch, E. Zuazua. Numerical approximation of the null controls for the heat equation through transmutation. J. Inverse Problems 26(8) (2010), doi:10.1088/0266-5611/26/8/085018.

[24] R.E.A.C. Paley, N. Wiener. Fourier Transforms in Complex Domains. AMS Colloq. Publ., Vol. 19, Amer. Math. Soc., New-York, 1934.

[25] M. Tucsnak, G. Weiss. Observation and Control for Operator Semigroups. Birkhuser Advanced Texts, Springer, Basel, 2009 .

[26] C.F. Weber. Analysis and solution of the ill-posed inverse heat conduction problem. Int. J. Heat Mass Transfer 24 (1981), 1783-92.

[27] R.M. Young. An Introduction to Nonharmonic Fourier Series. Academic Press, New-York, 1980. 